\title{
Effortless quasi-interpolation in hierarchical spaces
}

\author{
Hendrik Speleers Carla Manni
}

Report TW647, May 2014

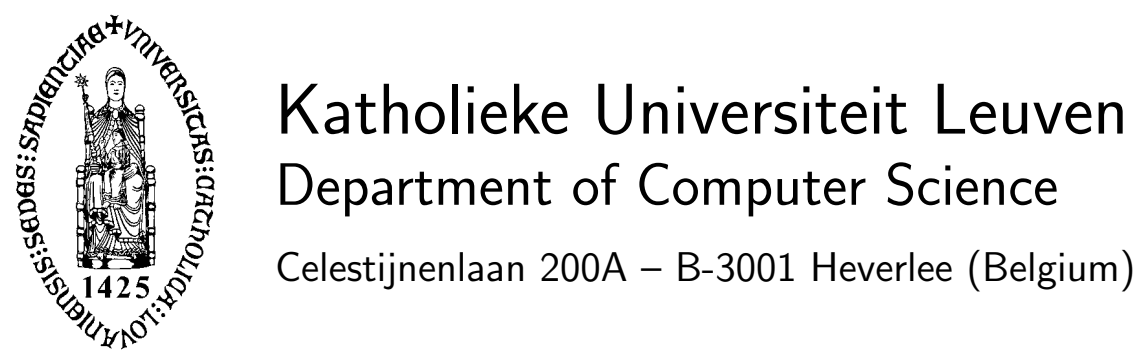




\title{
Effortless quasi-interpolation in hierarchical spaces
}

\author{
Hendrik Speleers Carla Manni \\ Report TW647, May 2014
}

Department of Computer Science, KU Leuven

\begin{abstract}
We present a general and simple procedure to construct quasi-interpolants in hierarchical spaces, which are composed of a hierarchy of nested spaces. The hierarchical quasi-interpolants are described in terms of the truncated hierarchical basis. Once for each level in the hierarchy a quasi-interpolant is selected in the corresponding space, the hierarchical quasi-interpolants are obtained without any additional manipulation. The main properties of the quasi-interpolants selected at each level are preserved in the hierarchical construction. In particular, hierarchical local projectors are constructed, and the local approximation order of the underling hierarchical space is investigated. The presentation is detailed for the truncated hierarchical B-spline basis, and we discuss its extension to a more general framework.
\end{abstract}

Keywords : Quasi-interpolation, hierarchical bases, local refinement, tensorproduct B-splines. 
Noname manuscript No.

(will be inserted by the editor)

\title{
Effortless quasi-interpolation in hierarchical spaces
}

\author{
Hendrik Speleers · Carla Manni
}

Received: date / Accepted: date

\begin{abstract}
We present a general and simple procedure to construct quasi-interpolants in hierarchical spaces, which are composed of a hierarchy of nested spaces. The hierarchical quasiinterpolants are described in terms of the truncated hierarchical basis. Once for each level in the hierarchy a quasi-interpolant is selected in the corresponding space, the hierarchical quasi-interpolants are obtained without any additional manipulation. The main properties of the quasi-interpolants selected at each level are preserved in the hierarchical construction. In particular, hierarchical local projectors are constructed, and the local approximation order of the underling hierarchical space is investigated. The presentation is detailed for the truncated hierarchical B-spline basis, and we discuss its extension to a more general framework.
\end{abstract}

Keywords Quasi-interpolation · Hierarchical bases · Local refinement · Tensor-product B-splines

Mathematics Subject Classification (2000) 41A15 - 65D07 - 65D17

\section{Introduction}

In many application areas, ranging from geometric modeling to numerical simulation, it is important to use function spaces that support local refinement in order to address local features in an effective way. Dealing with local refinement is usually not so difficult in one-dimensional problems. However, it can be an arduous task in bivariate and multivariate applications, where the complexity of the discretization can be prohibitive. Additionally, the considered spaces are often required to have a good approximation power and a low dimension. Nestedness of these spaces is also an interesting property because it ensures that successive levels of discretization improve the accuracy of the approximation. Spline spaces,

H. Speleers

University of Leuven, Department of Computer Science

Celestijnenlaan 200A, B-3001 Leuven, Belgium

E-mail: hendrik.speleers@cs.kuleuven.be

C. Manni

University of Roma 'Tor Vergata', Department of Mathematics

Via della Ricerca Scientifica, 00133 Roma, Italy

E-mail: manni@mat.uniroma2.it 
in all the accepted meanings of the word, are very popular function spaces in applications, see e.g. $[5,9,13,27,41]$.

Besides the structure of the space, a good basis to represent its elements is an essential ingredient for designing efficient and stable algorithms. Usually, a good spline basis possesses the following properties:

- the basis elements have local - possibly minimum - support, so that the influence of any basis function is as local as possible;

- the basis is stable, so that a small perturbation of the coefficients has a small influence on the corresponding spline function and vice versa;

- the basis forms a convex partition of unity, which is a crucial property for geometric design and also ensures numerical stability.

In the bivariate (and multivariate) setting, the most common (polynomial) spline structures are tensor-product splines and splines on triangulations. Tensor-product splines are a very simple and elegant multivariate extension of univariate splines, and they are equipped with a basis possessing all the above properties: the tensor-product B-spline basis, see e.g. [41]. A main drawback is that the tensor-product structure prevents any possibility of local refinement.

Local refinement is inherently supported by spline spaces defined over general triangulations. However, in this case the structure of smooth spaces is often quite involved. In general, the dimension of such a space is not completely understood and the construction of a suitable basis is an arduous task. There are only results for particular choices of degree and smoothness, see [27] and references therein. Using triangulations endowed with a macro-structure can be of some help to analyze the corresponding spline spaces [27] and to construct bases with the above properties $[15,44,45,46]$. On the other hand, it can be quite toilsome to built sequences of (locally refined) nested spline spaces based on such macro-structures.

In the literature there exist several alternative approaches to construct spline spaces which allow local refinements. Examples are T-splines [2,43], LR-splines [16], polynomial splines over T-meshes [29,42], and hierarchical splines [18,20,21].

T-splines and LR-splines form a convex partition of unity, but they are not always linearly independent. Linear independence is not so relevant in the context of geometric design, but it is imperative for certain simulation paradigms like isogeometric analysis [13]. Particular subclasses and algorithms to detect and avoid linear dependence were recently identified in both cases, see $[2,16,30]$ and references therein. However, the related refinement procedures can be quite complex, especially for high-dimensional applications. On the other hand, polynomial spline spaces over T-meshes possess similar strong and weak points as spline spaces over triangulations.

In this paper we focus on hierarchical splines because they provide a flexible framework coupled with a remarkable intrinsic simplicity. Hierarchical B-splines are defined in terms of a hierarchy of locally refined meshes, reflecting different levels of refinement. They were introduced in [18] as an accumulation of tensor-product B-splines with nested knot vectors. Applications of the hierarchical approach can be found in $[18,22,38,50]$. The original set of hierarchical B-splines in [18] is not linearly independent and does not form a partition of unity, but the problem of linear independence has been solved in [24,25]. In addition, a quasi-interpolant that achieves the optimal local approximation order has been introduced in [25] as well.

An alternative basis for the same hierarchical space has been proposed in [20] and is called truncated hierarchical B-spline (THB-spline) basis. This basis forms a convex parti- 
tion of unity, and its elements are more locally supported than those of the classical hierarchical basis. Moreover, the THB-spline basis possesses some other interesting properties, see [21]. Under mild assumptions on the knot configurations, this basis is strongly stable with respect to the supremum norm. This means that the constants to be considered in the stability analysis of the basis do not depend on the number of hierarchical levels. It is also worth to mention that the truncation procedure preserves the coefficients of a function represented with respect to the underlying basis considered at a certain level in the hierarchy.

The construction of truncated hierarchical bases is not confined to tensor-product polynomial spline spaces. It has also been investigated in [47] for hierarchical spline spaces over Powell-Sabin triangulations, and in [21] for a broad class of hierarchical spaces. In this general setting, the truncated hierarchical bases maintain properties like convex partition of unity, stability, and preservation of coefficients.

In this paper we exploit the above properties of truncated hierarchical bases to obtain a general and very simple procedure for the construction of quasi-interpolants in hierarchical spaces. Thanks to the preservation of coefficients, the construction is basically effortless. It is sufficient to consider a quasi-interpolant in each space associated with a particular level in the hierarchy, which will be referred to as a one-level quasi-interpolant. Then, the coefficients of the proposed hierarchical quasi-interpolant are nothing else than a proper subset of the coefficients of the one-level quasi-interpolants. No additional manipulations are required. Important properties - like polynomial reproduction - of the one-level quasiinterpolants are preserved in the hierarchical construction. It is easy to construct hierarchical local projectors, and so to provide dual bases. We also investigate the local approximation order of the hierarchical quasi-interpolants.

The neat and general construction of the presented hierarchical quasi-interpolants is a consequence of the nice properties of the truncated hierarchical bases. It makes these bases and the corresponding spaces even more attractive for applications where local refinement is of interest.

For an easier reading, we elaborate the construction of hierarchical quasi-interpolants, the analysis of the hierarchical space, and the study of its local approximation power in terms of THB-splines. However, the procedure is completely general and can be applied to any set of bases (and corresponding spaces) that fits into the broad framework defined in [21].

The remaining of the paper is organized as follows. In Section 2 we recall the definition and main properties of THB-splines, and in the next section we give a characterization of the space they span. Section 4 is devoted to the construction of hierarchical quasi-interpolants, and in Section 5 we discuss their local approximation power. Section 6 briefly describes how the results in the previous sections can be extended to the general framework defined in [21]. We present several examples of hierarchies of spaces and bases that fit into this framework. In Section 7 we detail a simple but effective construction of hierarchical quasi-interpolants which are local projectors onto the THB-spline space. Their effectiveness is illustrated with two numerical experiments. We end in Section 8 with some concluding remarks.

\section{THB-splines}

Let $D$ be a hyper-rectangle in $\mathbb{R}^{d}$. We consider a nested sequence of tensor-product $d$-variate spline function spaces defined on $D$,

$$
\mathbb{V}^{0} \subset \mathbb{V}^{1} \subset \mathbb{V}^{2} \subset \ldots
$$



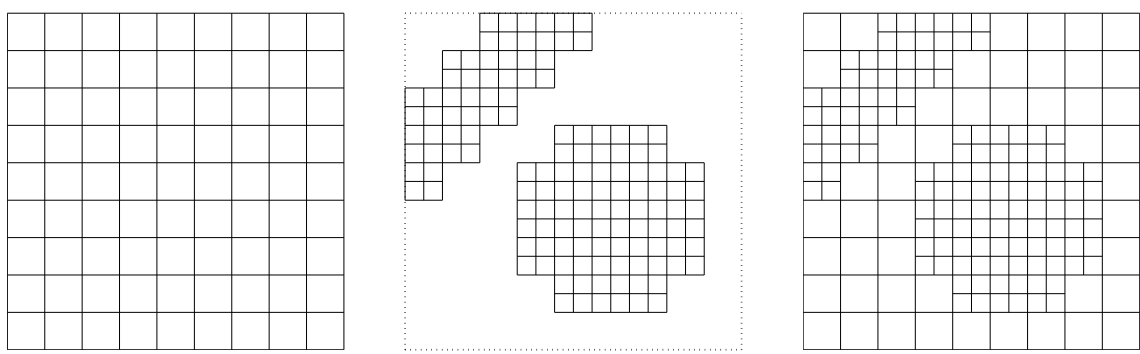

Fig. 1 A sequence of two-dimensional subsets $\Omega^{0} \supseteq \Omega^{1}$, together with their cells, used to build a hierarchical spline space. Left: $\Omega^{0}$; center: $\Omega^{1}$; right: the corresponding hierarchical mesh.

Any element of $\mathbb{V}^{\ell}$ is a piecewise polynomial defined over a partition of $D$ consisting of hyper-rectangles, which will be called cells of level $\ell$. Let $N_{\ell}$ be the dimension of $\mathbb{V}^{\ell}$, we denote by

$$
\mathscr{B}^{\ell}:=\left\{B_{i, \ell}, i=1, \ldots, N_{\ell}\right\}
$$

the normalized tensor-product B-spline basis of $\mathbb{V}^{\ell}$. It is well known (see e.g. [5,41]) that these basis functions are locally linearly independent, they have local support, they are nonnegative, and they form a partition of unity. Moreover, for any level $\ell$, each basis function in $\mathscr{B}^{\ell}$ can be expressed as a linear combination of basis functions in $\mathscr{B}^{\ell+1}$ with nonnegative coefficients. We will refer to this property as the two-scale relations with only nonnegative coefficients between bases of consecutive levels.

In addition to the spaces $\mathbb{V}^{\ell}$ and the corresponding bases $\mathscr{B}^{\ell}$, we consider a nested sequence of closed subsets of $D$,

$$
\Omega^{0} \supseteq \Omega^{1} \supseteq \Omega^{2} \supseteq \ldots
$$

where each $\Omega^{\ell}$ is the union of a selection of cells of level $\ell$. We assume that there exists $n \in \mathbb{N}$, such that

$$
\Omega^{n}:=\emptyset .
$$

Because of (4), the sequence in (3) is basically a finite one, which will be denoted by

$$
\boldsymbol{\Omega}_{n}:=\left\{\Omega^{0}, \Omega^{1}, \ldots, \Omega^{n-1}\right\} .
$$

We will refer to $\boldsymbol{\Omega}_{n}$ as the hierarchy of subsets of $D$ of depth $n$. Moreover, the collection of the corresponding cells in the hierarchy of the considered meshes will be shortly referred to as the hierarchical mesh of depth $n$. Note that a hierarchical mesh is not a partition of the domain because it consists of overlapping cells, in contrast to the conventional concept of (one-level) mesh. We also remark that each $\Omega^{\ell}$ is not necessarily a hyper-rectangle and can have several connected components. Figure 1(left-center) shows a sequence of twodimensional subsets $\Omega^{0} \supseteq \Omega^{1}$, together with their cells. The corresponding hierarchical mesh is depicted in Figure 1(right).

Finally, given a function $f$ defined on $D$, we denote by $\operatorname{supp}(f)$ the intersection of the support of $f$ with $\Omega^{0}$.

Given a sequence of spaces and bases as in (1)-(2) and a hierarchy of subsets as in (5), we can now construct the corresponding set of so-called hierarchical basis functions as follows. We first take all the basis elements in $\mathscr{B}^{0}$ whose support overlaps $\Omega^{0}$. Then, we apply an iterative procedure which selects at each level $\ell$ all the basis functions obtained in 
the previous step whose support is not entirely contained in $\Omega^{\ell}$ and all the basis functions in $\mathscr{B}^{\ell}$ whose support is entirely contained in $\Omega^{\ell}$. More precisely, we state the following definition, see $[20,21,50]$.

Definition 1 The hierarchical set of basis functions $\mathscr{H}_{\boldsymbol{\Omega}_{n}}$ associated to a hierarchy of subsets of $D$ as in (5) is recursively constructed as follows:

i) $\mathscr{H}^{0}:=\left\{B_{i, 0} \in \mathscr{B}^{0}: \operatorname{supp}\left(B_{i, 0}\right) \neq \emptyset\right\}$;

ii) for $\ell=0, \ldots, n-2$ :

$$
\mathscr{H}^{\ell+1}:=\mathscr{H}_{C}^{\ell+1} \cup \mathscr{H}_{F}^{\ell+1},
$$

where

$$
\begin{aligned}
& \mathscr{H}_{C}^{\ell+1}:=\left\{B_{i, j} \in \mathscr{H}^{\ell}: \operatorname{supp}\left(B_{i, j}\right) \nsubseteq \Omega^{\ell+1}\right\}, \\
& \mathscr{H}_{F}^{\ell+1}:=\left\{B_{i, \ell+1} \in \mathscr{B}^{\ell+1}: \operatorname{supp}\left(B_{i, \ell+1}\right) \subseteq \Omega^{\ell+1}\right\} ;
\end{aligned}
$$

iii) $\mathscr{H}_{\boldsymbol{\Omega}_{n}}:=\mathscr{H}^{n-1}$.

Note that by (1) and the construction of the set of functions $\mathscr{H}^{\ell}$ we easily see that

$$
\left\langle\mathscr{H}^{\ell}\right\rangle \subseteq\left\langle\mathscr{H}^{\ell+1}\right\rangle
$$

where $\langle\mathscr{H}\rangle$ stands for the span of the functions in the set $\mathscr{H}$. Let

$$
\mathbb{S}_{\boldsymbol{\Omega}_{n}}:=\left\langle B_{i, j}: B_{i, j} \in \mathscr{H}_{\boldsymbol{\Omega}_{n}}\right\rangle
$$

be the space spanned by the set of functions constructed in Definition 1 . We refer to it as the hierarchical space associated to $\boldsymbol{\Omega}_{n}$.

Since the elements in $\mathscr{B}^{\ell}$ are locally linearly independent, Definition 1 provides a set of linearly independent functions, see [50]. Therefore, the elements in $\mathscr{H}_{\boldsymbol{\Omega}_{n}}$ form a basis of $\mathbb{S}_{\boldsymbol{\Omega}_{n}}$, which will be referred to as the hierarchical basis of $\mathbb{S}_{\boldsymbol{\Omega}_{n}}$. The elements of the hierarchical basis are obviously nonnegative but they do not form a partition of unity.

For each $\ell=0, \ldots, n-1$, let $I_{\ell, \Omega_{n}}$ be the set of indices of the elements in $\mathscr{B}^{\ell}$ belonging to $\mathscr{H}_{\boldsymbol{\Omega}_{n}}$, i.e.,

$$
I_{\ell, \boldsymbol{\Omega}_{n}}:=\left\{i: B_{i, \ell} \in \mathscr{B}^{\ell} \cap \mathscr{H}_{\boldsymbol{\Omega}_{n}}\right\} .
$$

From Definition 1 it follows that

$$
I_{\ell, \boldsymbol{\Omega}_{n}}=\left\{i: B_{i, \ell} \in \mathscr{B}^{\ell}, \operatorname{supp}\left(B_{i, \ell}\right) \cap D^{\ell} \neq \emptyset, \operatorname{supp}\left(B_{i, \ell}\right) \subseteq \Omega^{\ell}\right\},
$$

where

$$
D^{\ell}:=\Omega^{\ell} \backslash \Omega^{\ell+1}
$$

By using (8), we can represent any element $s \in \mathbb{S}_{\boldsymbol{\Omega}_{n}}$ as

$$
s=\sum_{\ell=0}^{n-1} \sum_{i \in I_{\ell, \Omega_{n}}} d_{i, \ell} B_{i, \ell}
$$

We now modify the elements in $\mathscr{H}_{\boldsymbol{\Omega}_{n}}$ in order to construct an alternative basis for $\mathbb{S}_{\boldsymbol{\Omega}_{n}}$ with enhanced properties (like smaller support, convex partition of unity, ... ). The construction is based on the so-called truncation procedure which will be detailed in the following. 
Let $s \in \mathbb{V}^{\ell} \subset \mathbb{V}^{\ell+1}$ be represented with respect to the tensor-product B-spline basis $\mathscr{B}^{\ell+1}$, i.e.

$$
s=\sum_{i=1}^{N_{\ell+1}} c_{i, \ell+1} B_{i, \ell+1},
$$

where $c_{i, k}$ denotes the coefficient of $s \in \mathbb{V}^{k}$ with respect to the basis element $B_{i, k} \in \mathscr{B}^{k}$. We define the truncation of $s$ at level $\ell+1$ as the sum of the terms appearing in (11) related to the basis functions whose support is not a subset of $\Omega^{\ell+1}$, i.e.,

$$
\operatorname{trunc}^{\ell+1}(s):=\sum_{i: \operatorname{supp}\left(B_{i, \ell+1}\right) \nsubseteq \Omega^{\ell+1}} c_{i, \ell+1} B_{i, \ell+1} .
$$

Due to (4), we have that $\operatorname{trunc}^{n}(s)=s$. By using successive truncations of the functions constructed in Definition 1, we can define a new set of basis functions of $\mathbb{S}_{\boldsymbol{\Omega}_{n}}$.

Definition 2 The truncated hierarchical set of basis functions $\mathscr{T}_{\boldsymbol{\Omega}_{n}}$ associated to a hierarchy of subsets of $D$ as in (5) is recursively constructed as follows:

i) $\mathscr{T}^{0}:=\left\{B_{i, 0} \in \mathscr{B}^{0}: \operatorname{supp}\left(B_{i, 0}\right) \neq \emptyset\right\}$;

ii) for $\ell=0, \ldots, n-2$ :

$$
\mathscr{T}^{\ell+1}:=\mathscr{T}_{C}^{\ell+1} \cup \mathscr{T}_{F}^{\ell+1},
$$

where

$$
\begin{aligned}
& \mathscr{T}_{C}^{\ell+1}:=\left\{\operatorname{trunc}^{\ell+1}\left(B_{i, j}^{\mathscr{T}}\right): B_{i, j}^{\mathscr{T}} \in \mathscr{T}^{\ell}, \operatorname{supp}\left(B_{i, j}^{\mathscr{T}}\right) \nsubseteq \Omega^{\ell+1}\right\}, \\
& \mathscr{T}_{F}^{\ell+1}:=\left\{B_{i, \ell+1} \in \mathscr{B}^{\ell+1}: \operatorname{supp}\left(B_{i, \ell+1}\right) \subseteq \Omega^{\ell+1}\right\}
\end{aligned}
$$

iii) $\mathscr{T}_{\boldsymbol{\Omega}_{n}}:=\mathscr{T}^{n-1}$.

We denote the elements of $\mathscr{T}_{\boldsymbol{\Omega}_{n}}$ by

$$
\left\{B_{i, \ell, \boldsymbol{\Omega}_{n}}^{\mathscr{T}}, i \in I_{\ell, \boldsymbol{\Omega}_{n}}, \ell=0, \ldots, n-1\right\},
$$

and will refer to them as the truncated hierarchical B-splines (THB-splines). They are obtained by a successive truncation of the basis functions in $\mathscr{H}_{\boldsymbol{\Omega}_{n}}$, i.e.,

$$
B_{i, \ell, \boldsymbol{\Omega}_{n}}^{\mathscr{T}}=\operatorname{Trunc}_{\boldsymbol{\Omega}_{n}}^{\ell+1}\left(B_{i, \ell}\right), \quad i \in I_{\ell, \boldsymbol{\Omega}_{n}}, \quad \ell=0, \ldots, n-1,
$$

where for any $s \in \mathbb{V}^{\ell} \subset \mathbb{V}^{\ell+1}, \ell=0, \ldots, n-1$,

$$
\operatorname{Trunc}_{\boldsymbol{\Omega}_{n}}^{\ell+1}(s):=\operatorname{trunc}^{n-1}\left(\operatorname{trunc}^{n-2}\left(\ldots\left(\operatorname{trunc}^{\ell+1}(s)\right) \ldots\right)\right) .
$$

According to [20], the THB-splines form an alternative basis of $\mathbb{S}_{\boldsymbol{\Omega}_{n}}$. They are nonnegative and sum up to one, so they form a convex partition of unity. Moreover, the truncation mechanism ensures that the THB-splines have the same or smaller support than in the case of the classical hierarchical basis.

Figure 2 illustrates an example in the univariate case where the hierarchical basis and the truncated hierarchical basis are compared on a hierarchical mesh of depth 2 . This hierarchical mesh is defined starting from two nested knot vectors with knots of multiplicities 4 at the two endpoints of the interval and single knots elsewhere. In this example, all the basis functions have degree 3 .

From (13) we see that any THB-spline $B_{i, \ell, \Omega_{n}}^{\mathscr{T}}$ corresponds to a basis function $B_{i, \ell} \in$ $\mathscr{B}^{\ell}$. This correspondence has an important consequence, namely the so-called property of preservation of coefficients. This means that the THB-spline representation preserves the coefficients of functions represented with respect to one of the bases $\mathscr{B}^{\ell}$. This property is stated more precisely in the following theorem (see [21, Theorem 12]). 

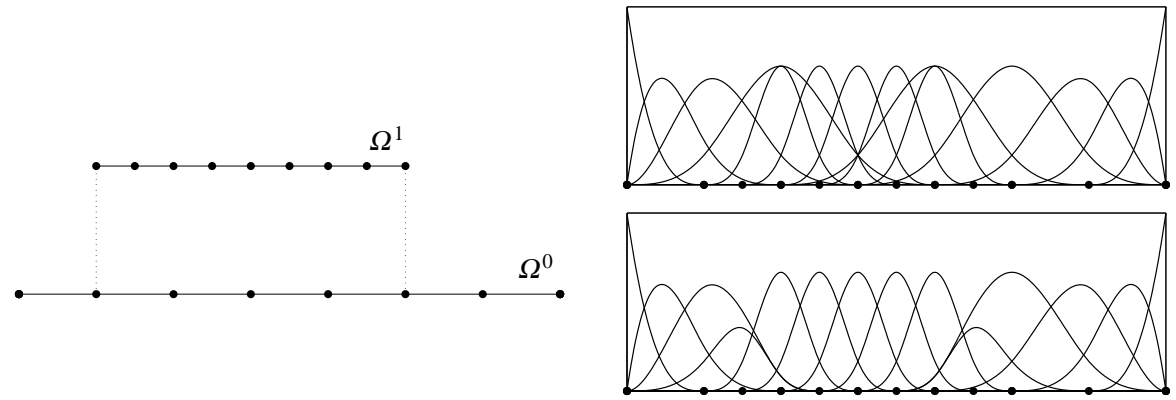

Fig. 2 Univariate classical and truncated hierarchical B-splines of degree 3 considered on a hierarchical mesh of depth 2. Left: hierarchy of subsets $\Omega^{0} \supseteq \Omega^{1}$; right: classical hierarchical B-splines (top) and truncated hierarchical B-splines (bottom).

Theorem 1 Let $\ell \in\{0, \ldots, n-1\}$ be given. Let $\left.s\right|_{D^{\ell}}$ be the restriction of $s \in \mathbb{S}_{\boldsymbol{\Omega}_{n}}$ to $D^{\ell}$, see (10), and consider its representation with respect to $\mathscr{B}^{\ell}$ and $\mathscr{T}_{\boldsymbol{\Omega}_{n}}$,

$$
\left.s\right|_{D^{\ell}}=\left.\sum_{j=1}^{N_{\ell}} c_{j, \ell} B_{j, \ell}\right|_{D^{\ell}}=\left.\sum_{k=0}^{n-1} \sum_{i \in I_{k, \boldsymbol{\Omega}_{n}}} c_{i, k}^{\mathscr{T}} B_{i, k, \boldsymbol{\Omega}_{n}}^{\mathscr{T}}\right|_{D^{\ell}} .
$$

Then,

$$
c_{i, \ell}^{\mathscr{T}}=c_{i, \ell}, \quad i \in I_{\ell, \boldsymbol{\Omega}_{n}} .
$$

Due to the nestedness of the spaces (1) we have the following corollary.

Corollary 1 Let $s \in \mathbb{V}^{0}$, and consider its representation with respect to $\mathscr{T}_{\boldsymbol{\Omega}_{n}}$ and $\mathscr{B}^{\ell}, \ell=$ $0, \ldots, n-1$,

$$
s=\sum_{j=1}^{N_{\ell}} c_{j, \ell} B_{j, \ell}=\sum_{k=0}^{n-1} \sum_{i \in I_{k, \boldsymbol{\Omega}_{n}}} c_{i, k}^{\mathscr{T}} B_{i, k, \boldsymbol{\Omega}_{n}}^{\mathscr{T}}
$$

Then,

$$
c_{i, \ell}^{\mathscr{T}}=c_{i, \ell}, \quad i \in I_{\ell, \boldsymbol{\Omega}_{n}}
$$

\section{The space $\mathbb{S}_{\boldsymbol{\Omega}_{n}}$}

In this section we characterize the elements of the space $\mathbb{S}_{\boldsymbol{\Omega}_{n}}$ spanned by the hierarchical basis $\mathscr{H}_{\boldsymbol{\Omega}_{n}}$ as well as by the truncated hierarchical basis $\mathscr{T}_{\boldsymbol{\Omega}_{n}}$.

We first define the restriction of the space $\left.\mathbb{V}^{\ell}\right|_{\gamma}$ as the span of the restrictions of all its basis functions $B_{i, \ell} \mid \mathrm{r}$, for any $\boldsymbol{\Upsilon} \subseteq D$. For the characterization of $\mathbb{S}_{\boldsymbol{\Omega}_{n}}$ proposed in the next theorem, we need to introduce the subsets

$$
R^{\ell}:=\Omega^{0} \backslash \Omega^{\ell+1}, \quad \ell=0, \ldots, n-1,
$$

so that from (3) we obtain that

$$
R^{\ell} \subseteq R^{\ell+1}
$$

and from (10) we have

$$
R^{\ell}=\cup_{k=0}^{\ell} \Omega^{k} \backslash \Omega^{k+1}=\cup_{k=0}^{\ell} D^{k}, \quad \ell=0, \ldots, n-1 .
$$


Theorem 2 Let

$$
\mathbb{W}_{\boldsymbol{\Omega}_{n}}:=\left\{s:\left.\left.s\right|_{R^{\ell}} \in \mathbb{V}^{\ell}\right|_{R^{\ell}}, \ell=0, \ldots, n-1\right\}
$$

then

$$
\mathbb{S}_{\boldsymbol{\Omega}_{n}}=\mathbb{W}_{\boldsymbol{\Omega}_{n}} \text { on } \Omega^{0} .
$$

Proof First we show that $\mathbb{S}_{\boldsymbol{\Omega}_{n}} \subseteq \mathbb{W}_{\boldsymbol{\Omega}_{n}}$. Because of the nestedness of the sequence of spaces (1), it is easy to see that for any $s \in \mathbb{S}_{\boldsymbol{\Omega}_{n}}$ we have

$$
\left.\left.s\right|_{R^{\ell}} \in \mathbb{V}^{\ell}\right|_{R^{\ell}}, \quad \ell=0, \ldots, n-1 .
$$

Now we will prove that $\mathbb{S}_{\boldsymbol{\Omega}_{n}} \supseteq \mathbb{W}_{\boldsymbol{\Omega}_{n}}$. Let us consider $s \in \mathbb{W}_{\boldsymbol{\Omega}_{n}}$. The restriction of $s$ to $R^{0}=D^{0}$ belongs to $\left.\mathbb{V}^{0}\right|_{R^{0}}$. Therefore, we can represent $\left.s\right|_{R^{0}}$ as a linear combination of the basis elements in $\mathscr{B}^{0}$ whose support intersects $R^{0}$, and by using (9) we have

$$
\left.s\right|_{R^{0}}=\left.\sum_{i: \operatorname{supp}\left(B_{i, 0}\right) \cap D^{0} \neq \emptyset} c_{i, 0} B_{i, 0}\right|_{R^{0}}=\left.\sum_{i \in I_{0, \Omega_{n}}} c_{i, 0} B_{i, 0}\right|_{R^{0}} .
$$

Then, we define the following functions on $\Omega^{0}$ :

$$
\begin{aligned}
s^{(0)} & :=\sum_{i \in I_{0, \Omega_{n}}} c_{i, 0} B_{i, 0}, \\
r^{(0)} & :=s-s^{(0)}
\end{aligned}
$$

so that

$$
\left.s^{(0)}\right|_{R^{0}}=\left.s\right|_{R^{0}},\left.\quad r^{(0)}\right|_{R^{0}}=0 .
$$

For any $g \in \mathbb{W}_{\boldsymbol{\Omega}_{n}}$ we have $\left.\left.g\right|_{R^{1}} \in \mathbb{V}^{1}\right|_{R^{1}}$, so we can express the restriction of $r^{(0)}$ to $R^{1}$ as follows,

$$
\begin{aligned}
\left.r^{(0)}\right|_{R^{1}} & =\left.\sum_{i: \operatorname{supp}\left(B_{i, 1}\right) \cap R^{1} \neq \emptyset} c_{i, 1} B_{i, 1}\right|_{R^{1}} \\
& =\left.\sum_{i: \operatorname{supp}\left(B_{i, 1}\right) \cap R^{0} \neq \emptyset} c_{i, 1} B_{i, 1}\right|_{R^{1}}+\left.\sum_{i: \operatorname{supp}\left(B_{i, 1}\right) \cap D^{1} \neq \emptyset, \operatorname{supp}\left(B_{i, 1}\right) \subseteq \Omega^{1}} c_{i, 1} B_{i, 1}\right|_{R^{1}} .
\end{aligned}
$$

Moreover, since $\left.r^{(0)}\right|_{R^{0}}=0$ and $R^{0} \subseteq R^{1}$, we know that

$$
\left.r^{(0)}\right|_{R^{0}}=\left.\sum_{i: \operatorname{supp}\left(B_{i, 1}\right) \cap R^{0} \neq \emptyset} c_{i, 1} B_{i, 1}\right|_{R^{0}}=0 .
$$

By the local linear independence of the basis elements in $\mathscr{B}^{1}$ we have that all the coefficients in the above sum are equal to 0 . Therefore, the first sum in (17) is zero, and by using (9), we obtain

$$
\left.r^{(0)}\right|_{R^{1}}=\left.\sum_{i: \operatorname{supp}\left(B_{i, 1}\right) \cap D^{1} \neq \emptyset, \operatorname{supp}\left(B_{i, 1}\right) \subseteq \Omega^{1}} c_{i, 1} B_{i, 1}\right|_{R^{1}}=\left.\sum_{i \in I_{1, \Omega_{n}}} c_{i, 1} B_{i, 1}\right|_{R^{1}} .
$$

Let $s^{(1)}, r^{(1)}$ be defined as

$$
\begin{aligned}
& s^{(1)}:=\sum_{i \in I_{1, \Omega_{n}}} c_{i, 1} B_{i, 1}, \\
& r^{(1)}:=r^{(0)}-s^{(1)}=s-s^{(0)}-s^{(1)},
\end{aligned}
$$


so that

$$
\left.s^{(1)}\right|_{R^{1}}=\left.r^{(0)}\right|_{R^{1}},\left.\quad r^{(1)}\right|_{R^{1}}=0
$$

As a consequence,

$$
\left.\left(s^{(1)}+s^{(0)}\right)\right|_{R^{1}}=\left.s\right|_{R^{1}} .
$$

We can repeat the same procedure for all the levels of the hierarchy by taking

$$
\begin{aligned}
s^{(\ell)} & :=\sum_{i \in I_{\ell, \Omega_{n}}} c_{i, \ell} B_{i, \ell}, \\
r^{(\ell)} & :=s-s^{(0)}-s^{(1)}-\ldots-s^{(\ell)},
\end{aligned}
$$

so that

$$
\left.r^{(\ell)}\right|_{R^{\ell}}=0,\left.\quad\left(s^{(\ell)}+\ldots+s^{(1)}+s^{(0)}\right)\right|_{R^{\ell}}=\left.s\right|_{R^{\ell}} .
$$

Since $\Omega^{n}=\emptyset$, we have $R^{n-1}=\Omega^{0}$. Hence, for $\ell=n-1$ the above equality reads as

$$
\left.s\right|_{\Omega^{0}}=\left.\sum_{\ell=0}^{n-1} s^{(\ell)}\right|_{\Omega^{0}}=\left.\sum_{\ell=0}^{n-1} \sum_{i \in I_{\ell, \boldsymbol{\Omega}_{n}}} c_{i, \ell} B_{i, \ell}\right|_{\Omega^{0}} \in \mathbb{S}_{\boldsymbol{\Omega}_{n}} .
$$

So, we conclude that $\mathbb{S}_{\boldsymbol{\Omega}_{n}}=\mathbb{W}_{\boldsymbol{\Omega}_{n}}$ on $\Omega^{0}$.

Note that any element $s \in \mathbb{W}_{\boldsymbol{\Omega}_{n}}$ satisfies

$$
\left.\left.S\right|_{D^{\ell}} \in \mathbb{V}^{\ell}\right|_{D^{\ell}}
$$

The global smoothness of the elements in $\mathbb{W}_{\boldsymbol{\Omega}_{n}}$ is determined by the smoothness of the spaces $\mathbb{V}^{\ell}$ in the sequence (1).

An interesting case is described in the following example.

Example 1 Let each $\mathbb{V}^{\ell}$ be a tensor-product spline space with degree $p$ in each coordinate and with maximal smoothness $C^{p-1}$ defined on $D$. Then,

$$
\mathbb{S}_{\boldsymbol{\Omega}_{n}} \subseteq\left\{s \in C^{p-1}\left(\Omega^{0}\right):\left.\left.s\right|_{D^{\ell}} \in \mathbb{V}^{\ell}\right|_{D^{\ell}}, \ell=0, \ldots, n-1\right\} .
$$

The relation between the space $\mathbb{S}_{\boldsymbol{\Omega}_{n}}$ and the space of piecewise polynomials with degree $p$ in each coordinate and with maximal smoothness has been investigated in [19] for the bivariate case. The trivariate case has been addressed in [3].

Assume we have two hierarchies of subsets of $D$, namely $\boldsymbol{\Omega}_{n}$ and $\widehat{\boldsymbol{\Omega}}_{\hat{n}}$ of depth $n$ and $\hat{n}$, respectively. We say that $\widehat{\boldsymbol{\Omega}}_{\hat{n}}$ enlarges $\boldsymbol{\Omega}_{n}$ if

$$
n \leq \hat{n}, \quad \Omega^{0}=\widehat{\Omega}^{0}, \quad \Omega^{\ell} \subseteq \widehat{\Omega}^{\ell}, \quad \ell=1, \ldots, n-1 .
$$

The next corollary states that the corresponding hierarchical spaces are nested as well. This has already been proved in [21, Proposition 6] in a different way.

Corollary 2 Let $\boldsymbol{\Omega}_{n}$ and $\widehat{\boldsymbol{\Omega}}_{\hat{n}}$ be two hierarchies of subsets of $D$ of depth $n$ and $\hat{n}$, respectively, such that $\widehat{\boldsymbol{\Omega}}_{\hat{n}}$ enlarges $\boldsymbol{\Omega}_{n}$. Then,

$$
\mathbb{S}_{\boldsymbol{\Omega}_{n}} \subseteq \mathbb{S}_{\widehat{\boldsymbol{\Omega}}_{\hat{n}}} \text { on } \Omega^{0}
$$

Proof Let $\widehat{R}^{\ell}:=\widehat{\Omega}^{0} \backslash \widehat{\Omega}^{\ell+1}$ and let $R^{\ell}$ be defined as in (14). From (18) it follows that $\widehat{R}^{\ell} \subseteq R^{\ell}$. Since the spaces in (1) are nested, the definition in (15) implies that $\mathbb{W}_{\boldsymbol{\Omega}_{n}} \subseteq \mathbb{W}_{\widehat{\boldsymbol{\Omega}}_{\hat{n}}}$, and Theorem 2 gives (19). 


\section{Quasi-interpolants in $\mathbb{S}_{\boldsymbol{\Omega}_{n}}$}

The term quasi-interpolation denotes a general approach to construct, with a low computational cost, efficient approximants to a given set of data or a given function. A quasiinterpolant (QI) is usually obtained as a linear combination of the elements of a suitable set of functions that form a convex partition of unity and possess a small local support. These properties ensure both numerical stability and local control of the constructed approximant. Quasi-interpolants in polynomial spline spaces are a common and powerful approximation tool, see e.g. [4, 6, 32,39].

In this section we focus on quasi-interpolation in the space $\mathbb{S}_{\boldsymbol{\Omega}_{n}}$. As stated in Section 2, the THB-splines form a convex partition of unity, whereas the elements in $\mathscr{H}_{\boldsymbol{\Omega}_{n}}$ do not. Moreover, THB-splines have in general a smaller (or the same) support than the corresponding elements in $\mathscr{H}_{\boldsymbol{\Omega}_{n}}$. Hence, we will construct QIs in the space $\mathbb{S}_{\boldsymbol{\Omega}_{n}}$ in terms of THB-splines as defined in Definition 2, see also (13). In addition, the THB-spline basis possesses the property of preservation of coefficients, see Theorem 1 . This allows an easy construction of QIs in $\mathbb{S}_{\boldsymbol{\Omega}_{n}}$, once a sequence of QIs in the spaces $\mathbb{V}^{\ell}, \ell=0, \ldots, n-1$, is given.

Let $f \in C\left(\Omega^{0}\right)$ be given. We now consider a sequence of one-level QIs, namely

$$
\mathfrak{Q}^{\ell}(f):=\sum_{i=1}^{N_{\ell}} \lambda_{i, \ell}(f) B_{i, \ell}, \quad \ell=0, \ldots, n-1,
$$

where $\lambda_{i, \ell}$ are suitable linear functionals on $C\left(\Omega^{0}\right)$. We say that $\lambda_{i, \ell}$ is supported on $\Lambda_{i, \ell}$ if

$$
\left.f\right|_{\Lambda_{i, \ell}}=0 \Rightarrow \lambda_{i, \ell}(f)=0 .
$$

Examples of interesting QIs of the form (20) can be found in [4, 6, 28, 32,39].

In order to construct a suitable QI in $\mathbb{S}_{\boldsymbol{\Omega}_{n}}$, it is sufficient to select as coefficient for each basis element $B_{i, \ell, \Omega_{n}}^{\mathscr{T}}$ the coefficient of the corresponding basis element in $\mathscr{B}^{\ell}$ in (20). More precisely, we set

$$
\mathfrak{Q}(f):=\sum_{\ell=0}^{n-1} \sum_{i \in I_{\ell, \boldsymbol{\Omega}_{n}}} \lambda_{i, \ell}(f) B_{i, \ell, \boldsymbol{\Omega}_{n}}^{\mathscr{T}} .
$$

We will refer to QIs of the form (22) as hierarchical QIs.

Given a set of degrees

$$
\boldsymbol{p}:=\left(p_{1}, \ldots, p_{d}\right)
$$

let $\mathbb{P}_{\boldsymbol{p}}$ be the space of tensor-product polynomials of degree $p_{i}$ in the $i$-th coordinate. When assuming $\mathbb{P}_{\boldsymbol{p}} \subset \mathbb{V}^{0}$ on $\Omega^{0}$, from (1) and (6) it follows that

$$
\mathbb{P}_{\boldsymbol{p}} \subset \mathbb{S}_{\boldsymbol{\Omega}_{n}} \text { on } \Omega^{0}
$$

We now describe QIs reproducing polynomials in $\mathbb{P}_{\boldsymbol{p}}$.

Theorem 3 Let $\mathfrak{Q}^{\ell}$ be a given sequence of QIs as in (20), and let $\mathfrak{Q}$ be the corresponding hierarchical QI as in (22). If

$$
\mathfrak{Q}^{\ell}(q)=q, \quad \forall q \in \mathbb{P}_{\boldsymbol{p}}, \quad \ell=0, \ldots, n-1,
$$

then

$$
\mathfrak{Q}(q)=q, \quad \forall q \in \mathbb{P}_{\boldsymbol{p}}
$$


Proof Let $q \in \mathbb{P}_{\boldsymbol{p}} \subset \mathbb{V}^{\ell}$, then it can be uniquely represented as a linear combination of the basis elements in $\mathscr{B}^{\ell}$,

$$
q=\sum_{i=1}^{N_{\ell}} c_{i, \ell} B_{i, \ell}
$$

and since $\mathfrak{Q}^{\ell}(q)=q$ we have $\lambda_{i, \ell}(q)=c_{i, \ell}$. On the other hand, $q \in \mathbb{S}_{\boldsymbol{\Omega}_{n}}$, so

$$
q=\sum_{\ell=0}^{n-1} \sum_{i \in I_{\ell, \Omega_{n}}} c_{i, \ell}^{\mathscr{T}} B_{i, \ell, \Omega_{n}}^{\mathscr{T}} .
$$

From Corollary 1 it follows

$$
c_{i, \ell}^{\mathscr{T}}=c_{i, \ell}=\lambda_{i, \ell}(q), \quad i \in I_{\ell, \Omega_{n}}, \quad \ell=0, \ldots, n-1,
$$

implying that $\mathfrak{Q}(q)=q$.

In the next theorem we present a sufficient condition to construct QIs which are projectors onto $\mathbb{S}_{\boldsymbol{\Omega}_{n}}$.

Theorem 4 Let $\mathfrak{Q}^{\ell}$ be a given sequence of QIs as in (20), and let $\mathfrak{Q}$ be the corresponding hierarchical QI as in (22). Assume

$$
\mathfrak{Q}^{\ell}(s)=s, \quad \forall s \in \mathbb{V}^{\ell}, \quad \ell=0, \ldots, n-1,
$$

and each $\lambda_{i, \ell}$ used in (22) is supported on $D^{\ell}$, see (10). Then,

$$
\mathfrak{Q}(s)=s, \quad \forall s \in \mathbb{S}_{\boldsymbol{\Omega}_{n}} .
$$

Proof Due to the linearity of the QI, it suffices to prove that

$$
\lambda_{i, \ell}\left(B_{j, k, \boldsymbol{\Omega}_{n}}^{\mathscr{T}}\right)=\delta_{i, j} \delta_{\ell, k}, \quad i \in I_{\ell, \boldsymbol{\Omega}_{n}}, \quad j \in I_{k, \boldsymbol{\Omega}_{n}}, \quad k, \ell=0, \ldots, n-1,
$$

where $\delta_{r, s}$ stands for the classical Kronecker delta. Let $i$ and $\ell$ be fixed. To prove (24) we consider three cases.

- Let $k>\ell$, then $\left.B_{j, k, \boldsymbol{\Omega}_{n}}^{\mathscr{T}}\right|_{D^{\ell}}=0$, see Definition 2 . Since $\lambda_{i, \ell}$ is only supported on $D^{\ell}$, it follows from (21) that $\lambda_{i, \ell}\left(B_{j, k, \boldsymbol{\Omega}_{n}}^{\mathscr{T}}\right)=0$.

- We now consider the case $k=\ell$. Since $\mathfrak{Q}^{\ell}$ is a projector onto $\mathbb{V}^{\ell}$, we have that $\lambda_{i, \ell}\left(B_{j, \ell}\right)=$ $\delta_{i, j}$. From the preservation of coefficients, see Theorem 1, we obtain

$$
\lambda_{i, \ell}\left(B_{j, \ell, \boldsymbol{\Omega}_{n}}^{\mathscr{T}}\right)=\delta_{i, j}, \quad i, j \in I_{\ell, \boldsymbol{\Omega}_{n}} .
$$

- Finally, let $k<\ell$. Any $\left.B_{j, k, \boldsymbol{\Omega}_{n}}^{\mathscr{T}}\right|_{D^{\ell}}$ can then be expressed as a linear combination of the basis elements in $\mathscr{B}^{\ell}$ restricted to $D^{\ell}$, namely

$$
\left.B_{j, k, \boldsymbol{\Omega}_{n}}^{\mathscr{T}}\right|_{D^{\ell}}=\left.\sum_{r=1}^{N_{\ell}} c_{r, \ell}^{j, k} B_{r, \ell}\right|_{D^{\ell}}
$$

Due to the definition of $B_{j, k, \boldsymbol{\Omega}_{n}}^{\mathscr{T}}$ and the preservation of coefficients, we have

$$
c_{r, \ell}^{j, k}=0, \quad \text { if } r \in I_{\ell, \Omega_{n}} .
$$

Thus, by the support restriction of $\lambda_{i, \ell}$, we have for $i \in I_{\ell, \Omega_{n}}$,

$$
\lambda_{i, \ell}\left(B_{j, k, \boldsymbol{\Omega}_{n}}^{\mathscr{T}}\right)=\sum_{r=1}^{N_{\ell}} c_{r, \ell}^{j, k} \lambda_{i, \ell}\left(B_{r, \ell}\right)=\sum_{r=1}^{N_{\ell}} c_{r, \ell}^{j, k} \delta_{i, r}=c_{i, \ell}^{j, k}=0 .
$$


Remark 1 The sequence of one-level QIs as in (20) considered in Theorem 4 has to satisfy constraints more restrictive than those in Theorem 3: for each level $\ell, \mathfrak{Q}^{\ell}$ has to be a projector onto $\mathbb{V}^{\ell}$ and each $\lambda_{i, \ell}, i \in I_{\ell, \boldsymbol{\Omega}_{n}}$, has to be supported in $D^{\ell}$. The former constraint connects the one-level QIs with the sequence of spaces $\mathbb{V}^{0}, \ldots, \mathbb{V}^{n-1}$ and has a similar counterpart in Theorem 3. The latter constraint links the same sequence of QIs with the hierarchy of subsets $\boldsymbol{\Omega}_{n}$. In Section 7 we describe a possible simple strategy to construct one-level QIs satisfying such constraints. However, once a sequence of QIs as in (20) satisfying the hypotheses of Theorem 4 is available, the construction of a hierarchical QI which is a projector onto $\mathbb{S}_{\boldsymbol{\Omega}_{n}}$ does not require additional efforts with respect to a hierarchical QI which just reproduces polynomials.

Remark 2 Let $\left\{\lambda_{i, \ell}\right\}$ be a set of linear functionals in (22) that provide a projector onto $\mathbb{S}_{\boldsymbol{\Omega}_{n}}$. Then, because of (24), it is a dual basis for $\mathscr{T}_{\boldsymbol{\Omega}_{n}}$.

The hierarchical QI defined in (22) can be interpreted as a telescopic approximant, where for each level an approximant of the residual is added. A similar telescopic approach was also considered in [25]. To show this, we define the following set of indices

$$
J_{\ell, \boldsymbol{\Omega}_{n}}:=\left\{i: B_{i, \ell} \in \mathscr{B}^{\ell}, \operatorname{supp}\left(B_{i, \ell}\right) \subseteq \Omega^{\ell}\right\} .
$$

Referring to (9), it is easy to see that $I_{\ell, \boldsymbol{\Omega}_{n}} \subseteq J_{\ell, \boldsymbol{\Omega}_{n}}$.

Theorem 5 Let $\mathfrak{Q}^{\ell}$ be a given sequence of QIs as in (20), and let $\mathfrak{Q}$ be the corresponding hierarchical QI as in (22). Assume

$$
\mathfrak{Q}^{\ell}(s)=s, \quad \forall s \in \mathbb{V}^{\ell}, \quad \ell=0, \ldots, n-1,
$$

then

$$
\mathfrak{Q}(f)=\sum_{\ell=0}^{n-1} f^{(\ell)}
$$

where

$$
f^{(\ell)}:=\sum_{i \in J_{\ell, \Omega_{n}}} \lambda_{i, \ell}\left(f-f^{(0)}-f^{(1)}-\ldots-f^{(\ell-1)}\right) B_{i, \ell}, \quad \ell=0, \ldots, n-1 .
$$

Proof Each quasi-interpolant $\mathfrak{Q}^{\ell}, \ell=0, \ldots, n-1$, is assumed to be a projector onto the space $\mathbb{V}^{\ell}$, and because of the nestedness of the spaces $\mathbb{V}^{\ell} \subset \mathbb{V}^{\ell+1}$, every basis function $B_{i, \ell}$ can be represented as

$$
B_{i, \ell}=\sum_{j=1}^{N_{\ell+1}} \lambda_{j, \ell+1}\left(B_{i, \ell}\right) B_{j, \ell+1} .
$$

By exploiting the definition of the truncated basis (13) and (26), we obtain

$$
f^{(0)}=\sum_{i \in J_{0, \boldsymbol{\Omega}_{n}}} \lambda_{i, 0}(f) B_{i, 0}=\sum_{i \in I_{0, \boldsymbol{\Omega}_{n}}} \lambda_{i, 0}(f) B_{i, 0, \boldsymbol{\Omega}_{n}}^{\mathscr{T}}+\sum_{i \in J_{0, \boldsymbol{\Omega}_{n}}} \lambda_{i, 0}(f)\left(\sum_{j \in J_{1, \boldsymbol{\Omega}_{n}}} \lambda_{j, 1}\left(B_{i, 0}\right) B_{j, 1}\right) .
$$

Moreover,

$$
\begin{aligned}
f^{(1)} & =\sum_{i \in J_{1, \boldsymbol{\Omega}_{n}}} \lambda_{i, 1}(f) B_{i, 1}-\sum_{i \in J_{1, \boldsymbol{\Omega}_{n}}} \lambda_{i, 1}\left(f^{(0)}\right) B_{i, 1} \\
& =\sum_{i \in J_{1, \boldsymbol{\Omega}_{n}}} \lambda_{i, 1}(f) B_{i, 1}-\sum_{i \in J_{1, \boldsymbol{\Omega}_{n}}}\left(\sum_{j \in J_{0, \boldsymbol{\Omega}_{n}}} \lambda_{j, 0}(f) \lambda_{i, 1}\left(B_{j, 0}\right)\right) B_{i, 1} .
\end{aligned}
$$


Hence,

$$
f^{(0)}+f^{(1)}=\sum_{i \in I_{0, \boldsymbol{\Omega}_{n}}} \lambda_{i, 0}(f) B_{i, 0, \boldsymbol{\Omega}_{n}}^{\mathscr{T}}+\sum_{i \in J_{1, \boldsymbol{\Omega}_{n}}} \lambda_{i, 1}(f) B_{i, 1}
$$

We now remark that from the truncation definition (12)-(13) it follows that $\lambda_{i, 2}\left(B_{j, 0, \Omega_{n}}^{\mathscr{T}}\right)=0$ for any $i \in J_{2, \boldsymbol{\Omega}_{n}}$ and $j \in I_{0, \boldsymbol{\Omega}_{n}}$, and so

$$
\sum_{i \in J_{2, \Omega_{n}}} \lambda_{i, 2}\left(B_{j, 0, \boldsymbol{\Omega}_{n}}^{\mathscr{T}}\right) B_{i, 2}=0, \quad \forall j \in I_{0, \boldsymbol{\Omega}_{n}} .
$$

By using similar arguments as before, we can write (27) as

$$
\begin{aligned}
& f^{(0)}+f^{(1)}= \\
& \quad \sum_{i \in I_{0, \boldsymbol{\Omega}_{n}}} \lambda_{i, 0}(f) B_{i, 0, \boldsymbol{\Omega}_{n}}^{\mathscr{T}}+\sum_{i \in I_{1, \boldsymbol{\Omega}_{n}}} \lambda_{i, 1}(f) B_{i, 1, \boldsymbol{\Omega}_{n}}^{\mathscr{T}}+\sum_{i \in J_{1, \boldsymbol{\Omega}_{n}}} \lambda_{i, 1}(f)\left(\sum_{j \in J_{2, \boldsymbol{\Omega}_{n}}} \lambda_{j, 2}\left(B_{i, 1}\right) B_{j, 2}\right),
\end{aligned}
$$

and by means of (27) and (28) we obtain

$$
\begin{aligned}
f^{(2)} & =\sum_{i \in J_{2, \boldsymbol{\Omega}_{n}}} \lambda_{i, 2}(f) B_{i, 2}-\sum_{i \in J_{2, \boldsymbol{\Omega}_{n}}} \lambda_{i, 2}\left(f^{(0)}+f^{(1)}\right) B_{i, 2} \\
& =\sum_{i \in J_{2, \boldsymbol{\Omega}_{n}}} \lambda_{i, 2}(f) B_{i, 2}-\sum_{i \in J_{2, \boldsymbol{\Omega}_{n}}}\left(\sum_{j \in J_{1, \boldsymbol{\Omega}_{n}}} \lambda_{j, 1}(f) \lambda_{i, 2}\left(B_{j, 1}\right)\right) B_{i, 2},
\end{aligned}
$$

resulting in

$$
f^{(0)}+f^{(1)}+f^{(2)}=\sum_{i \in I_{0, \boldsymbol{\Omega}_{n}}} \lambda_{i, 0}(f) B_{i, 0, \boldsymbol{\Omega}_{n}}^{\mathscr{T}}+\sum_{i \in I_{1, \boldsymbol{\Omega}_{n}}} \lambda_{i, 1}(f) B_{i, 1, \boldsymbol{\Omega}_{n}}^{\mathscr{T}}+\sum_{i \in J_{2, \boldsymbol{\Omega}_{n}}} \lambda_{i, 2}(f) B_{i, 2} .
$$

By iterating over all levels in the hierarchy and repeating the same arguments, we get the relation (25).

From Theorem 5 one can derive the representation of the proposed QI in (22) in terms of the classical hierarchical basis as defined in Definition 1 . This conversion can be easily done by means of the knot insertion formula for B-splines.

\section{Approximation properties}

In this section we investigate the local approximation power of the space $\mathbb{S}_{\boldsymbol{\Omega}_{n}}$ in the supremum norm. Let $f \in C\left(\Omega^{0}\right)$, and let $\Upsilon$ be a subset of $\Omega^{0}$. We use the following notation,

$$
\|f\|:=\sup _{\boldsymbol{x} \in \Omega^{0}}|f(\boldsymbol{x})|, \quad\|f\|_{\Upsilon}:=\sup _{\boldsymbol{x} \in \Upsilon}|f(\boldsymbol{x})| .
$$

For a given quasi-interpolant $\mathfrak{Q}$, we denote by $\|\mathfrak{Q}\|$ the usual induced norm, namely

$$
\|\mathfrak{Q}\|:=\sup _{\|f\|=1}\|\mathfrak{Q} f\|
$$

Since the basis $\mathscr{T}_{\boldsymbol{\Omega}_{n}}$ forms a convex partition of unity, for any QI of the form (22) it is easy to see that

$$
\|\mathfrak{Q}\| \leq \sup _{\ell=0, \ldots, n-1 ; i \in I_{\ell, \Omega_{n}}}\left\|\lambda_{i, \ell}\right\|_{\Lambda_{i, \ell}}=: C_{\mathfrak{Q}}
$$

where $\left\|\lambda_{i, \ell}\right\|_{\Lambda_{i, \ell}}$ stands for the induced norm of the linear functional $\lambda_{i, \ell}$ on its support $\Lambda_{i, \ell}$, see (21). Finally, we denote by $\operatorname{diam}(\Psi)$ the diameter of the set $\Psi$, and by $\operatorname{conv}(\Psi)$ the convex hull of $\Psi$.

With some standard arguments we obtain the following result. 
Theorem 6 Let a hierarchical quasi-interpolant $\mathfrak{Q}$ as in (22) be given, such that $C_{\mathfrak{Q}}$ in (29) is bounded. Let $\Upsilon$ be a cell, we set

$$
\Lambda_{\Upsilon}:=\operatorname{conv}\left(\bigcup_{(i, \ell): \operatorname{supp}\left(B_{i, \ell, \Omega_{n}}^{\mathscr{T}}\right) \cap \Upsilon \neq \emptyset} \Lambda_{i, \ell} \cup \Upsilon\right)
$$

where $\Lambda_{i, \ell}$ are the supports defined in (21). If $f \in C^{p+1}\left(\Lambda_{\Upsilon}\right)$, and

$$
\left.\mathfrak{Q}(q)\right|_{\Upsilon}=\left.q\right|_{\Upsilon}, \quad \forall q \in \mathbb{P}_{\boldsymbol{p}}, \quad \boldsymbol{p}:=(p, \ldots, p),
$$

then

$$
\|f-\mathfrak{Q}(f)\|_{\Upsilon} \leq C\left(\operatorname{diam}\left(\Lambda_{\Upsilon}\right)\right)^{p+1}\left(1+C_{\mathfrak{Q}}\right) \sum_{|\boldsymbol{\alpha}|=p+1}\left\|D^{\boldsymbol{\alpha}} f\right\|_{\Lambda_{\Upsilon}},
$$

where $C$ is a constant independent of $f$ and $\operatorname{diam}\left(\Lambda_{\Upsilon}\right)$.

Proof Thanks to (31), for any polynomial $q \in \mathbb{P}_{\boldsymbol{p}}$, we have

$$
\|f-\mathfrak{Q}(f)\|_{\Upsilon}=\|f-q+q-\mathfrak{Q}(f)\|_{\Upsilon} \leq\|f-q\|_{\Upsilon}+\|\mathfrak{Q}(f-q)\|_{\Upsilon} .
$$

Moreover, from (22) and from the convex partition of unity property of the basis $\mathscr{T}_{\boldsymbol{\Omega}_{n}}$, we have

$$
\|\mathfrak{Q}(f-q)\|_{\Upsilon}=\sup _{\boldsymbol{x} \in \Upsilon}\left|\sum_{(i, \ell): \operatorname{supp}\left(B_{i, \ell, \boldsymbol{\Omega}_{n}}^{\mathscr{T}}\right) \cap \Upsilon \neq \emptyset} \lambda_{i, \ell}(f-q) B_{i, \ell, \boldsymbol{\Omega}_{n}}^{\mathscr{T}}(\boldsymbol{x})\right| \leq C_{\mathfrak{Q}}\|f-q\|_{\Lambda_{\Upsilon}} .
$$

Therefore,

$$
\|f-\mathfrak{Q}(f)\|_{r} \leq\left(1+C_{\mathfrak{Q}}\right)\|f-q\|_{\Lambda_{r}},
$$

and the bound (32) follows from the approximation properties of the space $\mathbb{P}_{\boldsymbol{p}}$.

Some remarks about the previous theorem are in order.

Remark 3 If the value $C_{\mathfrak{Q}}$ does not depend on $\operatorname{diam}\left(\Lambda_{\Upsilon}\right)$, then (32) provides the optimal local approximation order. This is the case when the "building blocks" in the construction of $\mathfrak{Q}$, namely $\mathfrak{Q}^{\ell}$ in (20), have a similar property, i.e., when

$$
C_{\mathfrak{Q}^{\ell}}:=\sup _{i=1, \ldots, N_{\ell}}\left\|\lambda_{i, \ell}\right\|_{\Lambda_{i, \ell}}
$$

does not depend on $\operatorname{diam}\left(\Lambda_{\Upsilon}\right)$. For a one-level QI with such a property, see e.g. [28].

Remark 4 Suppose the spaces $\mathbb{V}^{\ell}$ in (1) are tensor-product $d$-variate spline spaces such that $\mathbb{P}_{\boldsymbol{p}^{\ell}} \subset \mathbb{V}^{\ell}, \boldsymbol{p}^{\ell}:=\left(p_{\ell}, \ldots, p_{\ell}\right)$. Then, thanks to the locality of the assumptions in Theorem 6 , our construction can provide hierarchical QIs with local approximation order $p_{\ell}+1$.

Remark 5 In practice it is of interest that $\operatorname{diam}\left(\Lambda_{\Upsilon}\right)$ is of the same order of $\operatorname{diam}(\Upsilon)$. To obtain this, we have to control both the supports of the linear functionals $\lambda_{i, \ell}$ and those of the basis elements $B_{i, \ell, \Omega_{n}}^{\mathscr{T}}$. The former supports can be fixed by a proper selection of the QIs in (20). There are several examples of efficient spline QIs where the support of $\lambda_{i, \ell}$ is contained in the one of $B_{i, \ell}$, see [28,32]. The latter supports depend on the hierarchies of the subsets (3) and of the spaces (1). It is possible to provide selection strategies for $\Omega^{\ell}$, $\ell=0, \ldots, n-1$, such that only basis elements $B_{i, \ell, \Omega_{n}}^{\mathscr{T}}$ corresponding to a given number of consecutive levels are supported on a given cell. For example, the strategy in [21, Appendix A] ensures that only basis elements corresponding to at most two consecutive levels $\ell-1, \ell$ are supported on a cell of level $\ell$ in $D^{\ell}$. This will be elaborated in more detail in Example 2 . 
Remark 6 Theorem 6 is not confined to cells and it still holds for any subset $\Psi$ of $\Omega^{0}$. If $\Psi$ is a large subset, then the straightforward application of (32) does not provide any useful information because $\operatorname{diam}\left(\Lambda_{\Psi}\right)$ is large as well. However, the bound can be improved as follows. Let $\Upsilon_{1}, \ldots, \Upsilon_{M}$ be a given set of cells such that $\Psi \subseteq \bigcup_{j=1}^{M} \Upsilon_{j}$, then

$$
\|f-\mathfrak{Q}(f)\|_{\Psi} \leq\left\{\max _{j=1, \ldots, M}\left(\operatorname{diam}\left(\Lambda_{\Upsilon_{j}}\right)\right)^{p+1}\right\} C\left(1+C_{\mathfrak{Q}}\right) \sum_{|\boldsymbol{\alpha}|=p+1}\left\|D^{\boldsymbol{\alpha}} f\right\|_{\Delta \Psi},
$$

where $\Delta \Psi:=\bigcup_{j=1}^{M} \Lambda_{\Upsilon_{j}}$.

Remark 7 Because of the form of our QI in (22) and the local support of the basis functions, it is natural to require that the support of $\lambda_{i, \ell}$ is in the neighborhood of the support of the corresponding basis function. This ensures a complete locality of the QI. It is well known that this is a golden rule to construct good QIs. The choice of the support of $\lambda_{i, \ell}$ can also be motivated by the classical structure of the error bounds in (32) and (34). First, in view of (30), the diameter of $\Lambda_{i, \ell}$ has to be small in order to obtain a small error bound (see also Remark 5). Second, any set $\Lambda_{i, \ell}$ involved in (30) must be close to $\Upsilon$ so that the difference between $f$ and $\mathfrak{Q}(f)$ on $\Upsilon$ is controlled by the behavior of $f$ in the neighborhood of $\Upsilon$. Finally, we recall that, in order to obtain a hierarchical projector, the support of $\lambda_{i, \ell}$ is also constrained by the hierarchy $\boldsymbol{\Omega}_{n}$, see Theorem 4 . However, this additional constraint can be easily satisfied under some mild assumptions on the hierarchy. We refer to Section 7 for a practical construction.

Finally, inspired by Remark 5, we apply Theorem 6 to a specific case of interest where we consider a hierarchy of nested uniform spline spaces and a restricted local refinement strategy.

Example 2 We consider a sequence of spaces as in (1) where $\mathbb{V}^{k}$ is a tensor-product $d$ variate spline space of degree $\boldsymbol{p}:=(p, \ldots, p)$ on a uniform grid with mesh size $h_{k}$, and $\mathbb{V}^{k}$ is obtained from $\mathbb{V}^{k-1}$ by dyadic refinement. Hence, we have $h_{k}=h_{k-1} / 2$. In addition, we define $h_{-1}:=h_{0}$ for notational convenience. To construct the hierarchy of subsets $\boldsymbol{\Omega}_{n}$ as in (5), we follow the strategy proposed in [21, Appendix A]: given the auxiliary subsets

$$
\omega^{k}=\left\{\boldsymbol{x} \in \Omega^{k} \mid \forall B_{i, k} \in \mathscr{B}^{k}: \boldsymbol{x} \in \operatorname{supp}\left(B_{i, k}\right) \Rightarrow \operatorname{supp}\left(B_{i, k}\right) \subseteq \Omega^{k}\right\}, \quad k=0, \ldots, n-1,
$$

we choose $\Omega^{k+1} \subseteq \omega^{k}$ for $k=0, \ldots, n-2$. Roughly speaking, this strategy ensures that $\Omega^{k+1}$ is "sufficiently far" from the boundary of $\Omega^{k}$. In this case, from [21] we know that only THB-splines of level $k-1$ and $k$ can have support intersecting $D^{k}$. Let us take the set $\Upsilon$ in Theorem 6 as a cell of a given level $\ell$ in $D^{\ell}$, and it will be denoted by $\Upsilon_{\ell}$. Finally, we consider a sequence of one-level QIs as in (20) satisfying (23) with $\lambda_{i, k}$ such that $\Lambda_{i, k} \subseteq \operatorname{supp}\left(B_{i, k}\right)$, see e.g. [28,32]. Due to our specific construction of the hierarchy, we have for $\ell \geq 1$,

$$
\begin{aligned}
& \Lambda_{r_{\ell}} \subseteq \operatorname{conv}\left(\bigcup_{(i, k): \operatorname{supp}\left(B_{i, k, \Omega_{n}}^{\mathscr{T}}\right) \cap r_{\ell} \neq \emptyset} \operatorname{supp}\left(B_{i, k}\right)\right) \\
& \quad \subseteq \operatorname{conv}\left(\bigcup_{k \in\{\ell-1, \ell\}} \bigcup_{i: \operatorname{supp}\left(B_{i, k}\right) \cap r_{\ell} \neq \emptyset} \operatorname{supp}\left(B_{i, k}\right)\right)=\operatorname{conv}\left(\bigcup_{i: \operatorname{supp}\left(B_{i, \ell-1}\right) \cap r_{\ell} \neq \emptyset} \operatorname{supp}\left(B_{i, \ell-1}\right)\right),
\end{aligned}
$$

and for $\ell=0$,

$$
\Lambda_{\Upsilon_{0}} \subseteq \operatorname{conv}\left(\bigcup_{i: \operatorname{supp}\left(B_{i, 0}\right) \cap \Upsilon_{0} \neq \emptyset} \operatorname{supp}\left(B_{i, 0}\right)\right)
$$


Hence, $\Lambda_{r_{\ell}}$ is contained in the hyper-cube in $\mathbb{R}^{d}$ consisting of $(2 p+1) \times \cdots \times(2 p+1)$ cells of level $\max \{0, \ell-1\}$, and centered at the cell of level $\max \{0, \ell-1\}$ containing $\Upsilon_{\ell}$. We denote this hyper-cube by $\mathbb{U}_{r_{\ell}}$. Moreover,

$$
\operatorname{diam}\left(\Lambda_{r_{\ell}}\right) \leq \sqrt{d}(2 p+1) h_{\ell-1}=2 \sqrt{d}(2 p+1) h_{\ell}
$$

Then, from Theorem 6 it follows that for any $f \in C^{p+1}\left(\mathbb{Q} r_{\ell}\right)$ the quasi-interpolant $\mathfrak{Q}$ as in (22) satisfies

$$
\|f-\mathfrak{Q}(f)\| r_{\ell} \leq C\left(h_{\ell}\right)^{p+1}\left(1+C_{\mathfrak{Q}}\right) \sum_{|\boldsymbol{\alpha}|=p+1}\left\|D^{\boldsymbol{\alpha}} f\right\|_{\mathbb{Z}_{\mathrm{r}_{\ell}}},
$$

where $C$ is a constant independent of $f$ and $h_{\ell}$.

\section{Beyond tensor-product polynomial B-splines}

Both the definition of the truncated hierarchical bases and the construction of the related QIs presented in the previous sections have been elaborated for tensor-product polynomial $\mathrm{B}$-splines due to their relevant interest. However, for both of them it is not necessary to use tensor-product polynomial B-splines as building blocks. They can be replaced by different sets of basis functions possessing similar properties. In this section we recall from [21] a more general framework for the construction of truncated hierarchical bases, and we show that all the previous results can be straightforwardly extended to it.

Let $D$ be a bounded domain in $\mathbb{R}^{d}$. We consider a nested sequence of spaces $\mathbb{V}^{\ell}$, where each of them is assumed to be a finite-dimensional subspace of the space $C(D)$. Each space $\mathbb{V}^{\ell}$ is spanned by a basis

$$
\mathscr{B}^{\ell}:=\left\{B_{i, \ell}, i=1, \ldots, N_{\ell}\right\}
$$

with the following properties.

(A1) The functions in $\mathscr{B}^{\ell}$ are locally linearly independent.

(A2) The functions in $\mathscr{B}^{\ell}$ have local support. Moreover, for each function $B_{i, \ell} \in \mathscr{B}^{\ell}$, the boundary of the support consists of a finite number of smooth arcs.

(A3) The functions in $\mathscr{B}^{\ell}$ are nonnegative.

(A4) The functions in $\mathscr{B}^{\ell}$ form a partition of unity.

(A5) There exists a two-scale relation with only nonnegative coefficients between $\mathscr{B}^{\ell}$ and $\mathscr{B}^{\ell+1}$.

In this general framework each basis $\mathscr{B}^{\ell}$ naturally leads to a collection of cells of level $\ell$ which tessellates the domain $D$, see [21]. A sequence of nested subsets (3) can then be considered where $\Omega^{\ell}$ is the union of a selection of cells of level $\ell$.

Whenever a sequence of spaces $\mathbb{V}^{\ell}$ spanned by bases $\mathscr{B}^{\ell}$ satisfying (A1-A5) is given, and a corresponding sequence of subsets $\Omega^{\ell}$ is selected, then we can follow the same steps as in Definition 2 to obtain a truncated hierarchical basis. This basis forms a convex partition of unity and possesses the property of preservation of coefficients, see [21]. Similarly, any sequence of suitable quasi-interpolants in the spaces $\mathbb{V}^{\ell}$ allows us to construct quasi-interpolants in the resulting hierarchical space by means of the procedure detailed in Section 4.

Beyond classical polynomial univariate B-splines and their tensor-product extension, several families of basis functions and their corresponding function spaces satisfy assumptions (A1-A5). We consider in the following some relevant examples. For all of them there exist in the literature quasi-interpolants of the form (20). Therefore, the strategy proposed 
in Section 4 can be easily applied and the corresponding results can be straightforwardly generalized.

Example 3 Box splines are a possible generalization to the multivariate setting of the concept of univariate B-splines with uniform knots. They can be defined by successive univariate convolutions with the characteristic function over the interval $[0,1)$ along given directions, see [7]. Hence, a box spline $M_{\Xi}$ in $\mathbb{R}^{d}$ is associated with a set of directions in $\mathbb{R}^{d}$, usually collected in a $d \times n$ matrix $\Xi, n \geq d$, with columns in $\mathbb{R}^{d} \backslash \mathbf{0}$. There is a well established theory for spaces spanned by integer translates of box splines, namely

$$
\left\{M_{\Xi}(.-\boldsymbol{j}), \quad \boldsymbol{j} \in \mathbb{Z}^{d}\right\},
$$

defined by directions in $\mathbb{Z}^{d}$ such that $\Xi$ has full rank. Assumptions (A2-A4) are satisfied by this set of functions. Moreover, their smoothness and the polynomial space contained in the space they span can be completely characterized by means of $\Xi$, see [7]. Suitable scaled versions of $M_{\Xi}$ and of its translates immediately provide a sequence of nested spaces as in (1). Such scaled versions of $M_{\Xi}(.-j)$ can be connected by means of a two-scale relation with only nonnegative coefficients, see (A5).

In view of assumption (A1), we recall that for the elements in the set (36) local linear independence is equivalent to (global) linear independence. Their linear independence can be characterized in a very elegant way in terms of the matrix $\Xi$, see [7, Theorem 57]. The functions in (36) are linearly independent if and only if each regular $d \times d$ submatrix $\bar{\Xi}$ of $\Xi$ is unimodular, i.e.,

$$
\operatorname{det}(\bar{\Xi}) \in\{1,-1\}
$$

A relevant example of linearly independent box splines are bivariate box splines defined on the three-directional mesh [7,27]. For such box splines, several quasi-interpolants which reproduce polynomials up to a certain degree can be found in the literature, see $[1,7,9,10$, $14,27]$ and references therein. For local quasi-interpolant projectors we also refer to [31]. Linearly independent box splines on more general lattices also result in bases satisfying assumptions (A1-A5). Their definition and some corresponding quasi-interpolants of the form (20) can be found in [23] and references therein.

Example 4 Powell-Sabin splines are bivariate quadratic $C^{1}$ splines defined over any given triangulation endowed with a particular 6-split macro-structure. For this spline space a Bspline-like basis has been constructed in [15], which satisfies assumptions (A1-A4). A sequence of nested spaces can be obtained by using suitable triadic refinements [49], and the corresponding B-spline-like bases satisfy (A5).

Besides the standard Hermite interpolant, several other quasi-interpolants of the form (20) have been proposed in the literature for Powell-Sabin splines, see $[35,40,46]$.

Truncated hierarchical bases ${ }^{1}$ based on Powell-Sabin B-splines have been previously presented in [47], and the local approximation power of the corresponding spaces has been investigated in [48] by exploiting hierarchical Hermite interpolants of the form (22).

Example 5 Piecewise Chebyshevian splines are splines with pieces taken from (different) extended Chebyshev spaces. Whenever piecewise Chebyshevian splines are good for de$s i g n^{2}$, see [37, Section 5] and [36], they possess a B-spline-like basis which satisfies assumptions (A1-A5). Interesting examples of nested piecewise Chebyshevian spline spaces

\footnotetext{
1 Note that the truncated hierarchical basis is called quasi-hierarchical basis in $[47,48]$.

2 Basically this means that the spline space contains constants and that an appropriate blossom can be constructed therein.
} 
good for design are the classical trigonometric/hyperbolic splines [8,41], the so-called generalized trigonometric/hyperbolic splines [8,11,26,51], and piecewise Müntz splines [37]. Some restrictions on the knot sequences can be necessary. Assumptions (A1-A5) also hold for tensor-products of these univariate splines which are defined on Cartesian grids.

Local quasi-interpolant projectors for classical and generalized trigonometric/hyperbolic splines of the form (20) can be found in [12,33,41]. A hierarchical framework based on generalized splines has already been described in [34].

\section{Numerical examples}

In this section we elaborate a simple example of hierarchical QIs as described in Section 4 where the underlying bases $\mathscr{B}^{\ell}$ consists of tensor-product B-splines. We also present some numerical results based on this construction in the case of THB-splines over uniform nested sequences of knots.

We now explain the construction of a possible hierarchical QI for any given function $f \in C(D)$. To this end, we first describe a quasi-interpolant

$$
\widetilde{\mathfrak{Q}}^{\ell}(f):=\sum_{i=1}^{N_{\ell}} \widetilde{\lambda}_{i, \ell}(f) B_{i, \ell}
$$

in the space $\mathbb{V}^{\ell}$ for a particular level $\ell$. It is based on the local projector procedure presented in [28]. With each basis element $B_{i, \ell} \in \mathscr{B}^{\ell}$ we associate the linear functional $\widetilde{\lambda}_{i, \ell}$ in (37) as follows. Let $\Theta_{i, \ell}$ be a subset of the support of $B_{i, \ell}$. We select a unisolvent set of $M_{i, \ell}$ interpolation points $\left\{\boldsymbol{x}_{j, i, \ell} \in \Theta_{i, \ell}, j=1, \ldots, M_{i, \ell}\right\}$, where $M_{i, \ell}$ is the number of the elements in $\mathscr{B}^{\ell}$ whose supports intersect $\Theta_{i, \ell}$. Then we solve the corresponding local interpolation problem

$$
\sum_{k: \operatorname{supp}\left(B_{k, \ell}\right) \cap \Theta_{i, \ell} \neq \emptyset} c_{k, \ell} B_{k, \ell}\left(\boldsymbol{x}_{j, i, \ell}\right)=f\left(\boldsymbol{x}_{j, i, \ell}\right), \quad j=1, \ldots, M_{i, \ell}
$$

Finally, $\tilde{\lambda}_{i, \ell}(f)$ is taken as the coefficient $c_{i, \ell}$ of $B_{i, \ell}$ in (38). Note that $\widetilde{\mathfrak{Q}}^{\ell}$ is a projector onto $\mathbb{V}^{\ell}$.

According to (22), the sequence of quasi-interpolants $\widetilde{\mathfrak{Q}}^{\ell}, \ell=0, \ldots, n-1$, leads to the hierarchical quasi-interpolant

$$
\widetilde{\mathfrak{Q}}(f):=\sum_{\ell=0}^{n-1} \sum_{i \in I_{\ell, \boldsymbol{\Omega}_{n}}} \widetilde{\lambda}_{i, \ell}(f) B_{i, \ell, \boldsymbol{\Omega}_{n}}^{\mathscr{T}},
$$

which ensures polynomial reproduction as described in Theorem 3 .

To obtain a projector onto $\mathbb{S}_{\boldsymbol{\Omega}_{n}}$, we need to select $\Theta_{i, \ell}$ in agreement with the sequence of the subsets (3). More precisely, according to Theorem 4, we look for linear functionals $\tilde{\lambda}_{i, \ell}$, $i \in I_{\ell, \boldsymbol{\Omega}_{n}}$, which are supported on $D^{\ell}$, see (10). Hence, we require that

$$
\Theta_{i, \ell} \subseteq D^{\ell}, \quad i \in I_{\ell, \Omega_{n}} .
$$

The above constraint can always be satisfied. Indeed, by the definition in (9) we have that $\operatorname{supp}\left(B_{i, \ell}\right) \cap D^{\ell} \neq \emptyset$ for any $i \in I_{\ell, \Omega_{n}}$.

In practice, a natural choice is to select $\Theta_{i, \ell}$ as a cell of level $\ell$ in the support of $B_{i, \ell}$. With such a selection the fulfillment of the constraint (40) requires some mild assumptions on the hierarchy $\boldsymbol{\Omega}_{n}$. For instance, (40) can be satisfied when the sets $\Omega^{k}$ in (3) are taken as 
the union of a selection of cells of level $k-1$, for $k \geq 1$. An example of such a hierarchical mesh is depicted in Figure 1. Note that this assumption on the sets $\Omega^{k}$ is usually satisfied because it is nothing else than a standard cell refinement. Moreover, selecting $\Theta_{i, \ell}$ as a cell of level $\ell$ results in some further benefits.

- From the implementation point of view, it is easy to find a cell of level $\ell$ which is in the support of the corresponding B-spline $B_{i, \ell}$ and satisfies (40). Indeed, it just requires to run over the few a priori known cells forming the support of $B_{i, \ell}$ and check (40). Once we have selected an appropriate cell, the coefficient $\widetilde{\lambda}_{i, \ell}$ can be immediately constructed without any further information about the hierarchy.

- A too small support of $\widetilde{\lambda}_{i, \ell}$ can incur numerical instabilities and a dangerous deterioration of the constructed quasi-interpolant. Because of the constraint (40), this can occur when $D^{\ell}$ is small. By selecting $\Theta_{i, \ell}$ as a cell of level $\ell$ we avoid such problems.

We now illustrate the use of the hierarchical quasi-interpolant $\widetilde{\mathfrak{Q}}$, with $\Theta_{i, \ell}$ selected as a cell of level $\ell$, by means of two numerical examples (Examples 6 and 7). In both examples, we choose the following setup. The THB-splines are constructed starting from a hierarchy of tensor-product B-splines of bidegree $\left(p_{1}, p_{2}\right)$ and maximal smoothness defined on uniform nested knot sequences. We have implemented two possible selections of the cell $\Theta_{i, \ell}$ in the support of the B-spline $B_{i, \ell}$ consisting of $\left(p_{1}+1\right) \times\left(p_{2}+1\right)$ cells of level $\ell$, locally indexed by $\left\{\left(j_{1}, j_{2}\right)\right\}_{j_{1}=0, j_{2}=0}^{p_{1}, p_{2}}$.

- Take $\Theta_{i, \ell}$ as the central cell of level $\ell$ in the support of $B_{i, \ell}$, with local cell index

$$
\left(\left\lfloor p_{1} / 2\right\rfloor,\left\lfloor p_{2} / 2\right\rfloor\right) \text {. }
$$

The corresponding one-level QI is a projector onto $\mathbb{V}^{\ell}$ but (40) is not necessarily satisfied. Therefore, the resulting hierarchical QI only guarantees polynomial reproduction (see Theorem 3), and is indicated by $\widetilde{\mathfrak{Q}}^{\mathrm{p}}$.

- Let $K_{i, \ell}$ be the set of local indices of admissible cells of level $\ell$ in the support of $B_{i, \ell}$, namely those that belong to $D^{\ell}$. If $\left(\left\lfloor p_{1} / 2\right\rfloor,\left\lfloor p_{2} / 2\right\rfloor\right) \in K_{i, \ell}$, then take $\Theta_{i, \ell}$ as the corresponding cell of level $\ell$. Otherwise, take $\Theta_{i, \ell}$ as one of the cells in the support of $B_{i, \ell}$ with local cell index

$$
\arg \min _{\left(j_{1}, j_{2}\right) \in K_{i, \ell}}\left(\min \left(\left|j_{1}-\left\lfloor p_{1} / 2\right\rfloor\right|,\left|j_{1}-\left\lceil p_{1} / 2\right\rceil\right|\right)+\min \left(\left|j_{2}-\left\lfloor p_{2} / 2\right\rfloor\right|,\left|j_{2}-\left\lceil p_{2} / 2\right\rceil\right|\right)\right) .
$$

It means that the selected cell $\Theta_{i, \ell}$ is one of the most central cells that satisfies (40). The resulting hierarchical QI is a projector (so it reproduces hierarchical splines, see Theorem 4), and is indicated by $\widetilde{\mathfrak{Q}}^{\mathrm{S}}$.

The corresponding unisolvent set of interpolation points required in (38) is taken as a uniform $\left(p_{1}+1\right) \times\left(p_{2}+1\right)$ tensor-product grid over the selected cell $\Theta_{i, \ell}$. Note that $M_{i, \ell}=$ $\left(p_{1}+1\right)\left(p_{2}+1\right)$ in this case.

Remark 8 The one-level QIs given in (37) which are used in the construction of $\widetilde{\mathfrak{Q}}^{\mathrm{p}}$ are very easy to compute, and do not require any information about the hierarchy of the locally refined meshes. We may say that the construction of $\widetilde{\mathfrak{Q}}^{\mathrm{p}}$ is truly effortless. On the other hand, as mentioned in Remark 1, in order to obtain a hierarchical spline projector (and so also $\widetilde{\mathfrak{Q}}^{\mathrm{S}}$ ), it is unavoidable that the construction of the corresponding one-level QIs needs to exploit knowledge about the local refinement. Of course, it is clear that for globally refined meshes $\widetilde{\mathfrak{Q}}^{\mathrm{p}}$ is equal to $\widetilde{\mathfrak{Q}}^{\mathrm{s}}$, so they are both projectors. 

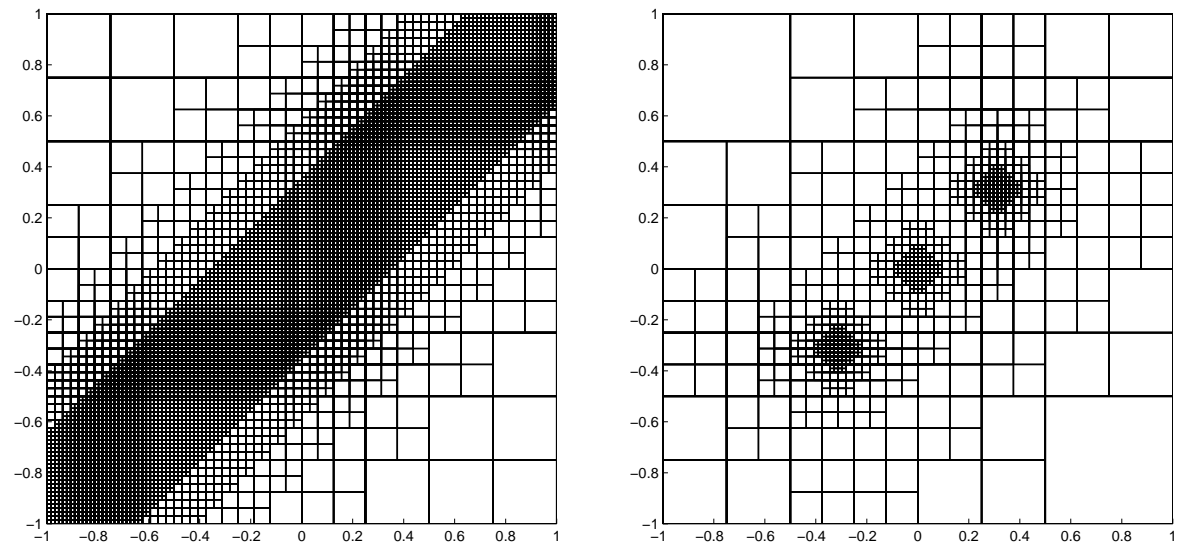

Fig. 3 Locally refined hierarchical meshes of depth 6. Left: Example 6; right: Example 7.

In Examples 6 and 7, we present several hierarchical quasi-interpolants of the form (39) computed on different sequences of hierarchical meshes of depths $n=1, \ldots, 6$ according to the above setup. In order to confirm the theoretical approximation order investigated in Section 5, we consider nested hierarchical spaces (7). We recall that nestedness of hierarchical spaces is ensured whenever sequences of enlarged hierarchies of subsets of $D$ are used, see Corollary 2. For all the mesh sequences, the coarsest mesh $(n=1)$ consists of $4 \times 4$ cells, and any cell of level $\ell$ is of size $h_{\ell}:=2^{-(\ell+1)}, \ell=0, \ldots, 5$. The maximum error is always computed on a uniform $150 \times 150$ grid.

Example 6 We approximate the function

$$
f(x, y)=\frac{\tanh (9 y-9 x)+1}{9}
$$

on the square $\Omega^{0}=[-1,1] \times[-1,1]$. This function is taken from [17] and simulates a sharp diagonal rise. We consider a sequence of hierarchical meshes of depths $n=1, \ldots, 6$, which are locally refined in the neighborhood of the diagonal rise. The final mesh of depth $n=6$ is depicted in Figure 3(left). The remaining meshes of depth $n \leq 5$ can be obtained by taking the first $n$ levels of the final mesh. We also consider a sequence of six uniform (globally refined) meshes. We then compute the hierarchical quasi-interpolants $\widetilde{\mathfrak{Q}}^{\mathrm{S}}(f)$ of bidegree $(p, p), p=2,3,4$ with maximal smoothness on both sequences of meshes, according to the method described above. The dimension of the corresponding spaces and the maximum errors are given in Table 1. We observe that the locally refined QIs obtain the same accuracy as the corresponding globally refined ones of the same depth, but of course with a lower dimension. For the sake of completeness, we also provide a comparison with discrete leastsquares approximations defined on the same locally refined meshes given a $150 \times 150$ grid of sampled data; they are far more expensive to compute because they require a global system to solve. Finally, as expected for a smooth function $f$, all the sequences of QIs present an optimal approximation order of $p+1$. Note that the higher degree splines have more difficulties to approximate accurately a step-like function on coarse meshes. 


\begin{tabular}{c|c|rr|rrr}
\hline & & \multicolumn{2}{c}{ global refinement } & \multicolumn{3}{c}{ local refinement } \\
bidegree & depth & dim & max error $\widetilde{\mathfrak{Q}}^{\mathrm{s}}$ & dim & max error $\widetilde{\mathfrak{Q}}^{\mathrm{s}}$ & max error LS \\
\hline$(2,2)$ & 1 & 36 & $7.434 \mathrm{e}-02$ & 36 & $7.434 \mathrm{e}-02$ & $4.744 \mathrm{e}-02$ \\
& 2 & 100 & $4.165 \mathrm{e}-02$ & 100 & $4.165 \mathrm{e}-02$ & $2.389 \mathrm{e}-02$ \\
& 3 & 324 & $1.000 \mathrm{e}-02$ & 264 & $1.000 \mathrm{e}-02$ & $5.836 \mathrm{e}-03$ \\
& 4 & 1156 & $1.280 \mathrm{e}-03$ & 754 & $1.280 \mathrm{e}-03$ & $7.125 \mathrm{e}-04$ \\
& 5 & 4356 & $1.025 \mathrm{e}-04$ & 2260 & $1.025 \mathrm{e}-04$ & $7.757 \mathrm{e}-05$ \\
& 6 & 16900 & $1.099 \mathrm{e}-05$ & 6120 & $1.099 \mathrm{e}-05$ & $9.175 \mathrm{e}-07$ \\
\hline$(3,3)$ & 1 & 49 & $4.691 \mathrm{e}-02$ & 49 & $4.691 \mathrm{e}-02$ & $4.038 \mathrm{e}-02$ \\
& 2 & 121 & $7.645 \mathrm{e}-02$ & 121 & $7.645 \mathrm{e}-02$ & $1.953 \mathrm{e}-02$ \\
& 3 & 361 & $1.287 \mathrm{e}-02$ & 289 & $1.287 \mathrm{e}-02$ & $3.763 \mathrm{e}-03$ \\
& 4 & 1225 & $4.515 \mathrm{e}-04$ & 775 & $4.515 \mathrm{e}-04$ & $2.387 \mathrm{e}-04$ \\
& 5 & 4489 & $1.124 \mathrm{e}-05$ & 2257 & $1.124 \mathrm{e}-05$ & $8.455 \mathrm{e}-06$ \\
& 6 & 17161 & $6.927 \mathrm{e}-07$ & 6037 & $6.927 \mathrm{e}-07$ & $9.814 \mathrm{e}-08$ \\
\hline$(4,4)$ & 1 & 64 & $1.730 \mathrm{e}-01$ & 64 & $1.730 \mathrm{e}-01$ & $3.461 \mathrm{e}-02$ \\
& 2 & 144 & $1.015 \mathrm{e}-01$ & 144 & $1.015 \mathrm{e}-01$ & $1.733 \mathrm{e}-02$ \\
& 3 & 400 & $2.945 \mathrm{e}-02$ & 316 & $2.945 \mathrm{e}-02$ & $3.129 \mathrm{e}-03$ \\
& 4 & 1296 & $4.710 \mathrm{e}-04$ & 798 & $4.710 \mathrm{e}-04$ & $1.343 \mathrm{e}-04$ \\
& 5 & 4624 & $1.028 \mathrm{e}-05$ & 2256 & $1.028 \mathrm{e}-05$ & $1.608 \mathrm{e}-06$ \\
& 6 & 17424 & $1.982 \mathrm{e}-07$ & 5956 & $2.036 \mathrm{e}-07$ & $1.373 \mathrm{e}-08$
\end{tabular}

Table 1 Dimensions and maximum errors of spline quasi-interpolants $\widetilde{\mathfrak{Q}}^{\mathrm{s}}$ of different degrees and maximal smoothness defined on globally and locally refined hierarchical meshes of different depths, see Example 6 . The maximum errors of the corresponding least-squares (LS) approximations are also provided.

\begin{tabular}{c|rr|rrr} 
& \multicolumn{2}{|c|}{ global refinement } & \multicolumn{4}{|c}{ local refinement } \\
depth & $\operatorname{dim}$ & max error $\widetilde{\mathfrak{Q}}^{\mathrm{s}}$ & $\operatorname{dim}$ & max error $\widetilde{\mathfrak{Q}} \mathrm{p}$ & max error $\widetilde{\mathfrak{Q}}^{\mathrm{s}}$ \\
\hline 1 & 36 & $5.686 \mathrm{e}-01$ & 36 & $5.686 \mathrm{e}-01$ & $5.686 \mathrm{e}-01$ \\
2 & 100 & $4.465 \mathrm{e}-01$ & 64 & $4.177 \mathrm{e}-01$ & $4.483 \mathrm{e}-01$ \\
3 & 324 & $2.575 \mathrm{e}-01$ & 112 & $2.636 \mathrm{e}-01$ & $2.575 \mathrm{e}-01$ \\
4 & 1156 & $1.472 \mathrm{e}-01$ & 190 & $1.472 \mathrm{e}-01$ & $1.472 \mathrm{e}-01$ \\
5 & 4356 & $5.955 \mathrm{e}-02$ & 456 & $5.955 \mathrm{e}-02$ & $5.955 \mathrm{e}-02$ \\
6 & 16900 & $2.156 \mathrm{e}-02$ & 600 & $3.177 \mathrm{e}-02$ & $2.156 \mathrm{e}-02$
\end{tabular}

Table 2 Dimensions and maximum errors of the biquadratic $C^{1}$ spline quasi-interpolants $\widetilde{\mathfrak{Q}}^{\mathrm{p}}$ and $\widetilde{\mathfrak{Q}}^{\mathrm{s}}$ defined on globally and locally refined hierarchical meshes of different depths, see Example 7.

Example 7 We approximate the following function composed of three peaks,

$$
\begin{aligned}
f(x, y)= & \frac{2}{3 \exp \left(\sqrt{(10 x-3)^{2}+(10 y-3)^{2}}\right)}+\frac{2}{3 \exp \left(\sqrt{(10 x+3)^{2}+(10 y+3)^{2}}\right)} \\
& +\frac{2}{3 \exp \left(\sqrt{(10 x)^{2}+(10 y)^{2}}\right)},
\end{aligned}
$$

on the square $\Omega^{0}=[-1,1] \times[-1,1]$, see [20]. We now consider the sequence of locally refined hierarchical meshes given in [20, Figure 6], and again a sequence of uniform (globally refined) meshes. The locally refined hierarchical mesh of depth $n=6$ is depicted in Figure 3(right). We consider biquadratic $C^{1}$ hierarchical spline spaces. Their dimensions and the maximum errors of the corresponding quasi-interpolants $\mathfrak{Q}^{\mathrm{p}}$ and $\widetilde{\mathfrak{Q}}^{\mathrm{s}}$ are shown in Table 2. Because of the low smoothness of $f$ it is not possible to obtain an approximation order three, but we observe that a comparable accuracy can be reached with a much smaller dimension thanks to the local refinements. On average, the projector $\widetilde{\mathfrak{Q}}^{\mathrm{S}}$ performs slightly better than the less complicated quasi-interpolant $\widetilde{\mathfrak{Q}}^{\mathrm{p}}$, see also Remark 8. For a comparison with the corresponding discrete least-squares approximations, we refer to [20]. 


\section{Conclusions}

In this paper we have presented a general and simple procedure to construct QIs in hierarchical spaces. Such spaces are composed of a hierarchy of nested spaces, in order to deal with local refinement in a straightforward way. The presented hierarchical QIs are described in terms of the truncated hierarchical basis, and their construction deeply benefits from many interesting properties of this basis, in particular the property of preservation of coefficients.

These hierarchical QIs are obtained without any additional manipulation, once a QI is selected in the space associated to each level in the hierarchy. Important properties - like polynomial reproduction - of the one-level QIs are preserved in the hierarchical construction. Hierarchical local projectors (and so dual bases) can be constructed easily. In addition, we have provided a characterization of the underlying hierarchical space, and we have analyzed its local approximation power.

We have detailed the construction of hierarchical QIs and their properties in terms of hierarchies of truncated tensor-product polynomial B-splines, the so-called THB-splines. Nevertheless, the procedure is completely general and can be applied to any set of bases that fits into the broad framework defined in [21]. Other examples are box splines on the threedirectional mesh and linearly independent box splines on more general lattices, PowellSabin B-splines with triadic refinement, and Chebyshevian B-splines good for design.

From $[20,21]$ we know that the truncated hierarchical basis has many attractive properties. The presented neat and completely general construction of hierarchical QIs makes the truncated hierarchical basis even more appealing for applications where local refinement is of interest.

An interesting generalization of the above results is to consider spaces spanned by a set of linearly dependent functions, e.g. the well-known case of box splines on the fourdirectional mesh. In this perspective, the above construction cannot be immediately extended due to the lack of linear independence and requires a deeper investigation.

\section{Acknowledgements}

This work was partially supported by the Research Foundation Flanders and by INdAMGNCS Gruppo Nazionale per il Calcolo Scientifico. We would like to thank Bert Jüttler (Johannes Kepler University, Linz) for fruitful discussions and for pointing out the idea of the telescopic interpretation.

\section{References}

1. Barrera, D., Ibáñez, M.J., Sablonnière, P., Sbibih, D.: Near-best quasi-interpolants associated with $H$ splines on a three-direction mesh. J. Comput. Appl. Math. 183, 133-152 (2005)

2. Beirão da Veiga, L., Buffa, A., Sangalli, G., Vázquez, R.: Analysis-suitable T-splines of arbitrary degree: definition, linear independence and approximation properties. Math. Models Methods Appl. Sci. 23, 1979-2003 (2013)

3. Berdinsky, D., Kim, T.-W., Bracco, C., Cho, D., Mourrain, B., Oh, M.-J., Kiatpanichgij, S.: Dimensions and bases of hierarchical tensor-product splines. J. Comput. Appl. Math. 257, 86-104 (2014)

4. de Boor, C.: Quasi interpolants and approximation power of multivariate splines. In: Dahmen, W., Gasca, M., and Micchelli, C.A. (eds.), Computation of Curves and Surfaces, pp. 313-345. Kluwer (1990)

5. de Boor, C.: A Practical Guide to Splines, Revised edition. Springer (2001)

6. de Boor, C., Fix, G.J.: Spline approximation by quasi-interpolants. J. Approx. Theory 8, 19-45 (1973)

7. de Boor, C., Höllig, K., Riemenschneider, S.: Box splines. Springer-Verlag (1993) 
8. Carnicer, J.M., Mainar, E., Peña, J.M.: Critical length for design purposes and Extended Chebyshev spaces. Constr. Approx. 20, 55-71 (2004)

9. Chui, C.K.: Multivariate Splines. CBMS-NSF Reg. Conf. Series in Appl. Math., vol. 54. SIAM, Philadelphia (1988)

10. Chui, C.K., Lai, M.-J.: A multivariate analog of Marsden's identity and a quasi-interpolation scheme. Constr. Approx. 3, 111-122 (1987)

11. Costantini, P., Lyche, T., Manni, C.: On a class of weak Tchebycheff systems. Numer. Math. 101, 333354 (2005)

12. Costantini, P., Manni, C., Pelosi, F., Sampoli, M.L.: Quasi-interpolation in isogeometric analysis based on generalized B-splines. Comput. Aided Geom. Design 27, 656-668 (2010)

13. Cottrell, J.A., Hughes, T.J.R., Bazilevs, Y.: Isogeometric Analysis: Toward Integration of CAD and FEA. John Wiley \& Sons (2009)

14. Dahmen, W., Micchelli, C.A.: On the approximation order from certain multivariate spline spaces. $J$. Austral. Math. Soc. Ser. B 26, 233-246 (1984)

15. Dierckx, P.: On calculating normalized Powell-Sabin B-splines. Comput. Aided Geom. Design 15, 61-78 (1997)

16. Dokken, T., Lyche, T., Pettersen, K.F.: Polynomial splines over locally refined box-partitions. Comput. Aided Geom. Design 30, 331-356 (2013)

17. Dyn N., Levin D., Rippa, S.: Data dependent triangulations for piecewise linear interpolation. IMA $J$. Numer. Anal. 10, 137-154 (1990)

18. Forsey, D.R., Bartels, R.H.: Hierarchical B-spline refinement. Comput. Graph. 22, 205-212 (1988)

19. Giannelli, C., Jüttler, B.: Bases and dimensions of bivariate hierarchical tensor-product splines. J. Comput. Appl. Math. 239, 162-178 (2013)

20. Giannelli, C., Jüttler, B., Speleers, H.: THB-splines: the truncated basis for hierarchical splines. Comput. Aided Geom. Design 29, 485-498 (2012)

21. Giannelli, C., Jüttler, B., Speleers, H.: Strongly stable bases for adaptively refined multilevel spline spaces. Adv. Comp. Math. 40, 459-490 (2014)

22. Greiner, G., Hormann, K.: Interpolating and approximating scattered 3D-data with hierarchical tensor product B-splines. In: Le Méhauté, A., Rabut, C., Schumaker, L.L. (eds.), Surface Fitting and Multiresolution Methods, pp. 163-172. Vanderbilt University Press (1997)

23. Kim, M., Peters, J.: Symmetric box-splines on root lattices. J. Comput. Appl. Math. 235, 3972-3989 (2011)

24. Kraft, R.: Adaptive and linearly independent multilevel B-splines. In: Le Méhauté, A., Rabut, C., Schumaker, L.L. (eds.), Surface Fitting and Multiresolution Methods, pp. 209-218. Vanderbilt University Press, Nashville (1997)

25. Kraft, R.: Adaptive und linear unabhängige Multilevel B-Splines und ihre Anwendungen. Ph.D. thesis, Universität Stuttgart (1998)

26. Kvasov, B.I., Sattayatham, P.: GB-splines of arbitrary order. J. Comput. Appl. Math. 104, 63-88 (1999)

27. Lai, M.-J, Schumaker, L.L.: Spline Functions on Triangulations. Cambridge U.P. (2007)

28. Lee, B.-G., Lyche, T., Mørken, K.: Some examples of quasi-interpolants constructed from local spline projectors. In: Lyche, T., Schumaker, L.L. (eds.), Mathematical Methods for Curves and Surfaces, Oslo 2000, pp. 243-252. Vanderbilt University Press (2001)

29. Li, X., Deng, J., Chen, F.: Polynomial splines over general T-meshes. Vis. Comput. 26, 277-286 (2010)

30. Li, X., Zheng, J., Sederberg, T.W., Hughes, T.J.R., Scott, M.A.: On linear independence of T-spline blending functions. Comput. Aided Geom. Design 29, 63-76 (2012)

31. Lyche, T., Manni, C., Sablonnière, P.: Quasi-interpolation projectors for box splines. J. Comput. Appl. Math. 221, 416-429 (2008)

32. Lyche, T., Schumaker, L.L.: Local spline approximation methods. J. Approx. Theory 15, 294-325 (1975)

33. Lyche, T., Schumaker, L.L., Stanley, S.: Quasi-interpolants based on trigonometric splines. J. Approx. Theory 95, 280-309 (1998)

34. Manni, C., Pelosi, F., Speleers, H.: Local hierarchical $h$-refinements in IgA based on generalized Bsplines. In: Floater, M.S., Lyche, T., Mazure, M.L., Mørken, K., Schumaker, L.L. (eds.), Mathematical Methods for Curves and Surfaces, Lecture Notes in Computer Science 8177, 341-363 (2014)

35. Manni, C., Sablonnière, P.: Quadratic spline quasi-interpolants on Powell-Sabin partitions. Adv. Comp. Math. 26, 283-304 (2007)

36. Mazure, M.L.: How to build all Chebyshevian spline spaces good for geometric design? Numer. Math. 119, 517-556 (2011)

37. Mazure, M.L.: On a new criterion to decide whether a spline space can be used for design. BIT Numer. Anal. 52, 1009-1034 (2012)

38. Rabut, C.: Locally tensor product functions. Numer. Algor. 39, 329-348 (2005) 
39. Sablonnière, P.: Recent progress on univariate and multivariate polynomial or spline quasi-interpolants. In: de Brujn, M.G., Mache, D.H., Szabadoz, J. (eds.), Trends and Applications in Constructive Approximation, ISNM Vol. 151, pp. 229-245. Birhäuser Verlag Basel (2005)

40. Sbibih, D., Serghini, A., Tijini, A.: Polar forms and quadratic spline quasi-interpolants on Powell-Sabin partitions. Appl. Numer. Math. 59, 938-958 (2009)

41. Schumaker, L.L.: Spline Functions: Basic Theory, Third edition. Cambridge U.P. (2007)

42. Schumaker, L.L., Wang, L.: Approximation power of polynomial splines on T-meshes. Comput. Aided Geom. Design 29, 599-612 (2012)

43. Sederberg, T.W., Zheng, J., Bakenov, A., Nasri, A.: T-splines and T-NURCCs. ACM Trans. Graphics 22, 477-484 (2003)

44. Speleers, H.: A normalized basis for reduced Clough-Tocher splines. Comput. Aided Geom. Design 27, 700-712 (2010)

45. Speleers, H.: Construction of normalized B-splines for a family of smooth spline spaces over PowellSabin triangulations. Constr. Approx. 37, 41-72 (2013)

46. Speleers, H.: Multivariate normalized Powell-Sabin B-splines and quasi-interpolants. Comput. Aided Geom. Design 30, 2-19 (2013)

47. Speleers, H., Dierckx, P., Vandewalle, S.: Quasi-hierarchical Powell-Sabin B-splines. Comput. Aided Geom. Design 26, 174-191 (2009)

48. Speleers, H., Dierckx, P., Vandewalle, S.: On the local approximation power of quasi-hierarchical PowellSabin splines. In: Dæhlen, M., Floater, M.S., Lyche, T., Merrien, J.L., Mørken, K., Schumaker, L.L. (eds.), Mathematical Methods for Curves and Surfaces, Lecture Notes in Computer Science 5862, 419433 (2010)

49. Vanraes, E., Windmolders, J., Bultheel, A., Dierckx, P.: Automatic construction of control triangles for subdivided Powel-Sabin splines. Comput. Aided Geom. Design 21, 671-682 (2004)

50. Vuong, A.-V., Giannelli, C., Jüttler, B., Simeon, B.: A hierarchical approach to adaptive local refinement in isogeometric analysis. Comput. Methods Appl. Mech. Engrg. 200, 3554-3567 (2011)

51. Wang, G., Fang, M.: Unified and extended form of three types of splines. J. Comput. Appl. Math. 216, 498-508 (2008) 